\title{
Further Results for General Financial Equilibrium Problems via Variational Inequalities
}

\author{
Annamaria Barbagallo ${ }^{1}$, Patrizia Daniele ${ }^{2}$, Mariagrazia Lorino ${ }^{2}$, \\ Antonino Maugeri ${ }^{2}$, Cristina Mirabella ${ }^{2}$ \\ ${ }^{1}$ Department of Mathematics and Applications "R. Caccioppoli", \\ University of Naples "Federico II", Naples, Italy \\ ${ }^{2}$ Department of Mathematics and Computer Science, \\ University of Catania, Catania, Italy \\ Email: annamaria.barbagallo@unina.it, daniele@dmi.unict.it, lorino@dmi.unict.it, \\ maugeri@dmi.unict.it, mirabella@dmi.unict.it
}

Received October 27, 2012; revised December 7, 2012; accepted December 21, 2012

\begin{abstract}
This paper is the sequel of the previous papers [1] and [2]. More precisely, we study the regularity of the solutions of the evolutionary variational inequality governing the general financial evolutionary problem. Specifically we obtain that such a solution is continuous and Lipschitz continuous with respect to time and we illustrate the achieved result through numerical examples. Moreover the numerical examples enables us to understand the behaviour of the financial equilibrium and the impact of the components of the model on the financial equilibrium.
\end{abstract}

Keywords: Financial Problem; Equilibrium Condition; Variational Inequality Formulation; Lagrange Variables; Deficit Formula; Balance Law; Liability Formula; Continuity; Lipschitz Continuity

\section{Introduction}

In the previous papers [1] and [2], a general equilibrium model of financial flows and prices is considered. The model is assumed evolving in time. The equilibrium conditions are considered in dynamic sense and the governing variational inequality formulation is presented. Precisely, the variational inequality we are working with is the following one:

$$
\begin{gathered}
\operatorname{Find}\left(x^{*}, y^{*}, r^{*}\right) \in \prod_{i=1}^{m} P_{i} \times \mathcal{R}: \\
\sum_{i=1}^{m} \int_{0}^{T}\left\{\sum_{j=1}^{n}\left[-\frac{\partial u_{i}\left(t, x_{i}^{*}(t), y_{i}^{*}(t)\right)}{\partial x_{i j}}-\left(1-\tau_{i j}(t)\right) r_{j}^{*}(t)\right] \times\left[x_{i j}(t)-x_{i j}^{*}(t)\right]\right. \\
\left.+\sum_{j=1}^{n}\left[-\frac{\partial u_{i}\left(t, x_{i}^{*}(t), y_{i}^{*}(t)\right)}{\partial y_{i j}}+\left(1-\tau_{i j}(t)\right) r_{j}^{*}(t)\left(1+h_{j}(t)\right)\right] \times\left[y_{i j}(t)-y_{i j}^{*}(t)\right]\right\} \mathrm{d} t \\
+\sum_{j=1}^{n} \int_{0}^{T} \sum_{i=1}^{m}\left\{\left(1-\tau_{i j}(t)\right)\left[x_{i j}^{*}(t)-\left(1+h_{j}(t)\right) y_{i j}^{*}(t)\right]+F_{j}(t)\right\} \times\left[r_{j}(t)-r_{j}^{*}(t)\right] \mathrm{d} t \geq 0, \\
\forall(x, y, r) \in \prod_{i=1}^{m} P_{i} \times \mathcal{R},
\end{gathered}
$$

where $P_{i}$ is the set of feasible assets and liabilities for each sector $i=1, \cdots, m$ given by

$$
\begin{aligned}
P_{i}= & \left\{\left(x_{i}, y_{i}\right) \in L^{2}\left([0, T], \mathbb{R}^{2 n}\right): \sum_{j=1}^{n} x_{i j}(t)=s_{i}(t), \text { a.e. in }[0, T],\right. \\
& \left.\sum_{j=1}^{n} y_{i j}(t)=l_{i}(t) \text { a.e. in }[0, T], \quad x_{i}(t) \geq 0, y_{i}(t) \geq 0, \text { a.e. in }[0, T]\right\},
\end{aligned}
$$


$s_{i}(t)$ is the total financial volume held by sector $i$ as assets, $l_{i}(t)$ is the total financial volume held by sector $i$ as liabilities, $-u_{i}\left(t, x_{i}(t), y_{i}(t)\right)$ is a measure of the risk of the financial agent, $\tau_{i j}(t)$ is the tax rate levied on sector $i$ 's net yield on financial instrument $j$, $h_{j}(t) \in L^{\infty}([0, T])$ is a nonnegative function, $F_{j}(t)$ is the portion of financial transaction per unit employed to cover the expenses of the financial institutions, $\mathcal{R}$ is the set of feasible instrument prices given by

$$
\begin{gathered}
\mathcal{R}=\left\{r \in L^{2}\left([0, T], \mathbb{R}^{n}\right): \underline{r}_{j}(t) \leq r_{j}(t) \leq \bar{r}_{j}(t),\right. \\
j=1, \cdots, n, \text { a.e. in }[0, T]\},
\end{gathered}
$$

where $\underline{r}$ and $\bar{r}$ are assumed to be in $L^{2}\left([0, T], \mathbb{R}^{n}\right)$.

Setting, for the sake of simplicity,

$$
\begin{gathered}
v=(x, y, r)=\left(\left(x_{i j}\right)_{\substack{i=1, \cdots, m \\
j=1, \cdots, n}},\left(y_{i j}\right)_{\substack{i=1, \cdots, m \\
j=1, \cdots, n}},\left(r_{j}\right)_{j=1, \cdots, n}\right) ; \\
A: L^{2}\left([0, T], \mathbb{R}^{2 m n+n}\right) \rightarrow L^{2}\left([0, T], \mathbb{R}^{2 m n+n}\right)
\end{gathered}
$$

defined as:

$$
\begin{aligned}
& A(t, v)=\left(\left[-\frac{\partial u_{i}(t, x, y)}{\partial x_{i j}}-\left(1-\tau_{i j}(t)\right) r_{j}(t)\right]_{\substack{i=1, \cdots, m \\
j=1, \cdots, n}},\right. \\
& {\left[-\frac{\partial u_{i}(t, x, y)}{\partial y_{i j}}+\left(1-\tau_{i j}(t)\right)\left(1+h_{j}(t)\right) r_{j}(t)\right]_{\substack{i=1, \cdots, m \\
j=1, \cdots, n}},} \\
& \left.\left[\sum_{i=1}^{m}\left(1-\tau_{i j}(t)\right)\left(x_{i j}(t)-\left(1+h_{j}(t)\right) y_{i j}(t)\right)\right]_{j=1, \cdots, n}\right) \\
& \mathbb{K}=\left\{v=(x, y, r) \in L^{2}\left([0, T], \mathbb{R}^{2 m n+n}\right):\right. \\
& x(t) \geq 0, y(t) \geq 0 \text {, a.e. in }[0, T], \\
& \sum_{j=1}^{n} x_{i j}(t)=s_{i}(t), \sum_{j=1}^{n} y_{i j}(t)=l_{i}(t), \text { a.e. in }[0, T] \text {, } \\
& \forall i=1, \cdots, m \text {, } \\
& \underline{r}(t) \leq r(t) \leq \bar{r}(t) \text {, a.e. in }[0, T]\},
\end{aligned}
$$

then variational inequality (1) becomes the problem:

Find $v^{*}=\left(x^{*}, y^{*}, r^{*}\right) \in \mathbb{K}:\left\langle A\left(t, v^{*}\right), v-v^{*}\right\rangle \geq 0$,

$\forall v \in \mathbb{K}$.

Variational inequality (1) proved to be a very useful tool which enables us to study the financial equilibrium of an economy evolving in time and in the previous papers [1] and [2] the authors provided an interesting and useful output as the deficit formula, a general balance law and the liability formula which could be of great importance for the theory of equilibrium problems evolving in time. For the reader's convenience we recall such important formulas:

\section{1) Deficit formula}

$$
\begin{aligned}
& \sum_{i=1}^{m}\left(1-\tau_{i j}(t)\right)\left[x_{i j}^{*}(t)-\left(1+h_{j}(t)\right) y_{i j}^{*}(t)\right] \\
& +F_{j}(t)+\rho_{j}^{(2) *}(t)=\rho_{j}^{(1) *}(t), \quad \forall j=1, \cdots, n,
\end{aligned}
$$

where $\rho_{j}^{(1) *}(t)$ and $\rho_{j}^{(2) *}(t)$ are the Lagrange variables associated to the price bounds:

$$
\begin{aligned}
& \rho_{j}^{(1) *}(t)\left(\underline{r}_{j}(t)-r_{j}^{*}(t)\right)=0, \quad \forall j=1, \cdots, n . \\
& \rho_{j}^{(2) *}(t)\left(r_{j}^{*}(t)-\bar{r}_{j}(t)\right)=0,
\end{aligned}
$$

The meaning of $\rho_{j}^{(1) *}(t)$ is that it represents the deficit per unit whereas $\rho_{j}^{(2) *}(t)$ is the positive surplus per unit;

2) Balance law

$$
\begin{aligned}
\sum_{i=1}^{m} l_{i}(t)= & \sum_{i=1}^{m} s_{i}(t)-\sum_{i=1}^{m} \sum_{j=1}^{n} \tau_{i j}(t)\left[x_{i j}^{*}(t)-y_{i j}^{*}(t)\right] \\
& -\sum_{i=1}^{m} \sum_{j=1}^{n}\left(1-\tau_{i j}(t)\right) h_{j}(t) y_{i j}^{*}(t) \\
& +\sum_{j=1}^{n} F_{j}(t)-\sum_{j=1}^{n} \rho_{j}^{(1) *}(t)+\sum_{j=1}^{n} \rho_{j}^{(2) *}(t) ;
\end{aligned}
$$

\section{3) Liability formula}

$$
\begin{aligned}
& \sum_{i=1}^{m} l_{i}(t)=\frac{1}{(1-\theta(t))(1+i(t))} \\
& \cdot\left\{(1-\theta(t)) \sum_{i=1}^{m} s_{i}(t)+\sum_{j=1}^{n} F_{j}(t)-\sum_{j=1}^{n} \rho_{j}^{(1) *}(t)+\sum_{j=1}^{n} \rho_{j}^{(2) *}(t)\right\},
\end{aligned}
$$

where $\theta(t)$ and $i(t)$ are the averages of $\tau_{i j}(t)$ and $h_{j}(t)$, namely $\theta(t)=\frac{\sum_{i=1}^{m} \sum_{j=1}^{n} \tau_{i j}(t)}{m n}$ and $i(t)=\frac{\sum_{j=1}^{n} h_{j}(t)}{n}$, respectively.

These suggested formulas could be of topical utility for the management of the world economy and to this aim in [2] the authors give some suggestions for the achievement of the world financial equilibrium and for finding the necessary way to follow in order to reach an improvement of the economy. It is worth reminding such suggestions:

Proposition 1.1 When the prices are minimal, namely they coincide with the floor prices, the economy collapses.

Proposition 1.2 Minimal prices imply the increase in the public debt.

Proposition 1.3 Minimal prices produce an economic recession. There is no incentive to the economic efforts.

Proposition 1.4 Even if it could be shocking, the 
development of the economy and of the employment results from an increase in the prices.

The value of these propositions can be realized by taking into account that the increase in prices indicated in Proposition 1.4 has been forecasted many months before (June 2011) it happened in our days.

Moreover, in [2] an "Evaluation Index", that we denoted by $E(t)$, has been introduced as a useful and simple tool for the evaluation of an economy, given by

$$
E(t)=\frac{\sum_{i=1}^{m} l_{i}(t)}{\sum_{i=1}^{m} \tilde{s}_{i}(t)+\sum_{j=1}^{n} \tilde{F}_{j}(t)},
$$

where we set

$$
\tilde{s}_{i}(t)=\frac{s_{i}(t)}{1+i(t)}, \quad \tilde{F}_{j}(t)=\frac{F_{j}(t)}{1+i(t)-\theta(t)-\theta(t) i(t)} .
$$

We remark that if $E(t)$ is greater or equal to 1 the evaluation of the financial equilibrium is positive (better if $E(t)$ is proximal to 1) whereas if $E(t)$ is less than 1 the evaluation of the financial equilibrium is negative.

The aim of this paper is to provide new theoretical and numerical results about solutions of financial equilibrium problems. In particular, we will prove a continuity result with respect to time of the solution, namely:

Theorem 1.1 Let $s \in C^{0}\left([0, T], \mathbb{R}^{m}\right)$, let $l \in C^{0}\left([0, T], \mathbb{R}^{n}\right)$, let $\underline{r}, \bar{r} \in C^{0}\left([0, T], \mathbb{R}_{+}^{n}\right)$ and let $A \in C^{0}\left([0, T], \mathbb{R}^{2 m n+n}\right)$ be a strongly monotone map, namely there exists $\alpha>0$ such that, for $t \in[0, T]$,

$$
\begin{aligned}
& \left\langle A\left(t, v_{1}\right)-A\left(t, v_{2}\right), v_{1}-v_{2}\right\rangle \geq \alpha\left\|v_{1}-v_{2}\right\|^{2}, \\
& \forall v_{1}, v_{2} \in \mathbb{R}^{2 m n+n} .
\end{aligned}
$$

Then variational inequality (1) admits a unique continuous solution.

Furthermore, we prove the following Lipschitz continuity result:

Theorem 1.2 Let $A \in C^{0}\left([0, T], \mathbb{R}^{2 m n+n}\right)$ be strongly monotone, namely there exists $\alpha>0$ such that, for $t \in[0, T]$,

$$
\begin{aligned}
& \left\langle A\left(t, v_{1}\right)-A\left(t, v_{2}\right), v_{1}-v_{2}\right\rangle \geq \alpha\left\|v_{1}-v_{2}\right\|^{2}, \\
& \forall v_{1}, v_{2} \in \mathbb{R}^{2 m n+n} ;
\end{aligned}
$$

Lipschitz continuous with respect to $v$, namely there exists $\beta>0$ such that, for $t \in[0, T]$

$$
\left\|A\left(t, v_{1}\right)-A\left(t, v_{2}\right)\right\| \leq \beta\left\|v_{1}-v_{2}\right\|, \forall v_{1}, v_{2} \in \mathbb{R}^{2 m n+n} ;
$$

and Lipschitz continuous with respect to $t$, namely there exists $M>0$ such that, for $t_{1}, t_{2} \in[0, T]$,

$$
\left\|A\left(t_{1}, v\right)-A\left(t_{2}, v\right)\right\| \leq M\|v\|\left|t_{1}-t_{2}\right|, \forall v \in \mathbb{R}^{2 m n+n} .
$$

Let $s, l:[0, T] \rightarrow \mathbb{R}^{m}$ be two Lipschitz continuous functions and let $\underline{r}, \bar{r}:[0, T] \rightarrow \mathbb{R}^{n}$ be two Lipschitz continuous functions. Then, the unique financial equilibrium solution $v^{*}=\left(x^{*}, y^{*}, r^{*}\right)$ is Lipschitz continuous in $[0, T]$. Moreover, let $t_{1} \neq t_{2}$, the following estimate holds:

$$
\frac{\left\|v^{*}\left(t_{2}\right)-v^{*}\left(t_{1}\right)\right\|^{2}}{\left|t_{2}-t_{1}\right|^{2}} \leq \gamma\left(\left\|v^{*}\right\|_{C^{0}\left([0, T], \mathbb{R}^{2 m n+n}\right)}^{2}+L^{2}\right),
$$

where $\gamma=\gamma(\alpha, \beta, M, T, L)$ and

$$
L=\sup _{\substack{t_{1}, t_{2} \in[0, T] \\ t_{1} \neq t_{2}}}\left\|\frac{P_{\mathbb{K}\left(t_{2}\right)}(z)-P_{\mathbb{K}\left(t_{1}\right)}(z)}{t_{2}-t_{1}}\right\| .
$$

The continuity and Lipschitz continuity of solutions to financial equilibrium problems is a very important property for applications. Indeed, it is fundamental to establish numerical approximate solutions. Such numerical solutions can be obtained making use of a modified version of the algorithms (see for instances [3-7]). It is worth remarking that the Lipschitz continuity allows us to calculate the error in approximating the solution.

In order to clearly illustrate theoretical results, some significant examples are provided and, in such a way, the impact that the components of the model have on the equilibrium are highlighted.

It is worth mensioning that even in this case variational inequalities are able to express the time-dependent equilibrium conditions. Then, applying delicate tools of nonlinear analysis (see [8-11]), it is possible to prove existence results and qualitative analysis. For other economic problems where the time plays an important role we refer to the papers devoted to the Walrasian equilibrium problem [12-15], to the oligopolistic market equilibrium problem $[16,17]$, to the weighted traffic equilibrium problem [18,19], and to [20].

The paper is organized as follows. In Section 2 we present the general financial model. In Section 3 we study the continuity results of the solution to the variational inequality which characterizes the financial model. In Section 4 we provide a Lipschitz continuity result for the solution. Finally, in Section 5 we propose a numerical examples from which we deduce that the solution, computed by means of the direct method (see [21]), is Lipschitz continuous.

\section{The Model}

We consider a financial economy consisting of $m$ sectors, with a typical sector denoted by $i$, and of $n$ instruments, with a typical financial instrument denoted by $j$, in the time interval $[0, T]$. Let $s_{i}(t)$ denote the total financial volume held by sector $i$ at time $t$ as assets, and let $l_{i}(t)$ be the total financial volume held 
by sector $i$ at time $t$ as liabilities. Then, unlike previous papers (see [22-27]), we allow markets of assets and liabilities to have different investments $s_{i}(t)$ and $l_{i}(t)$, respectively. Since we are working in the presence of uncertainty and of risk perspectives, the volumes $s_{i}(t)$ and $l_{i}(t)$ held by each sector cannot be considered stable with respect to time and may decrease or increase depending on unfavorable or favorable economic conditions. At time $t$, we denote the amount of instrument $j$ held as an asset in sector $i$ 's portfolio by $x_{i j}(t)$ and the amount of instrument $j$ held as a liability in sector $i$ 's portfolio by $y_{i j}(t)$. The assets and liabilities in all the sectors are grouped into the matrices

$$
x(t)=\left[\begin{array}{c}
x_{1}(t) \\
\vdots \\
x_{i}(t) \\
\vdots \\
x_{m}(t)
\end{array}\right]=\left[\begin{array}{ccccc}
x_{11}(t) & \ldots & x_{1 j}(t) & \ldots & x_{1 n}(t) \\
\vdots & \ddots & \vdots & \ddots & \vdots \\
x_{i 1}(t) & \ldots & x_{i j}(t) & \ldots & x_{i n}(t) \\
\vdots & \ddots & \vdots & \ddots & \vdots \\
x_{m 1}(t) & \ldots & x_{m j}(t) & \ldots & x_{m n}(t)
\end{array}\right]
$$

and

$$
y(t)=\left[\begin{array}{c}
y_{1}(t) \\
\vdots \\
y_{i}(t) \\
\vdots \\
y_{m}(t)
\end{array}\right]=\left[\begin{array}{ccccc}
y_{11}(t) & \ldots & y_{1 j}(t) & \ldots & y_{1 n}(t) \\
\vdots & \ddots & \vdots & \ddots & \vdots \\
y_{i 1}(t) & \ldots & y_{i j}(t) & \ldots & y_{i n}(t) \\
\vdots & \ddots & \vdots & \ddots & \vdots \\
y_{m 1}(t) & \ldots & y_{m j}(t) & \ldots & y_{m n}(t)
\end{array}\right] .
$$

We denote the price of instrument $j$ held as an asset at time $t$ by $r_{j}(t)$ and the price of instrument $j$ held as a liability at time $t$ by $\left(1+h_{j}(t)\right) r_{j}(t)$, where $h$ is a nonnegative function defined into $[0, T]$ and belonging to $L^{\infty}([0, T])$. We introduce the term $h_{j}(t)$ because the prices of liabilities are generally greater than or equal to the prices of assets so that we can describe, in a more realistic way, the behaviour of the markets for which the liabilities are more expensive than the assets. In such a way, this paper appears as an improvement in various directions of the previous ones [22-27]. We group the instrument prices held as assets into the vector

$r(t)=\left[r_{1}(t), r_{2}(t), \cdots, r_{i}(t), \cdots, r_{n}(t)\right]^{\mathrm{T}}$ and the instrument prices held as liabilities into the vector

$$
\begin{aligned}
& (1+h(t)) r(t) \\
= & {\left[\left(1+h_{1}(t)\right) r_{1}(t),\left(1+h_{2}(t)\right) r_{2}(t), \cdots,\right.} \\
& \left.\left(1+h_{i}(t)\right) r_{i}(t), \cdots,\left(1+h_{n}(t)\right) r_{n}(t)\right]^{\mathrm{T}}
\end{aligned}
$$

In our problem the prices of each instrument appear as unknown variables. Under the assumption of perfect competition, each sector will behave as if it has no influence on the instrument prices or on the behaviour of the other sectors.
In order to express the time-dependent equilibrium conditions by means of an evolutionary variational inequality, we choose as a functional setting the very general Lebesgue space $L^{2}\left([0, T], \mathbb{R}^{p}\right)$. Then, the set of feasible assets and liabilities for each sector $i=1, \cdots, m$, is $P_{i}$.

Now, in order to improve the model of competitive financial equilibrium described in [1], we consider the possibility of policy interventions in the financial equilibrium and incorporate them in form of taxes and price controls.

To this aim, denote the ceiling price associated with instrument $j$ by $\bar{r}_{j}$ and the nonnegative floor price associated with instrument $j$ by $\underline{r}_{j}$, with $\bar{r}_{j}(t)>\underline{r}_{j}(t)$, a.e. in $[0, T]$. The meaning of the constraint $r_{j}(t) \leq r_{j}(t)$ a.e. in $[0, T]$ is that to each investor a minimal price $\underline{r}_{j}$ for the assets held in the instrument $j$ is guaranteed, whereas each investor is requested to pay for the liabilities not less than the minimal price $\left(1+h_{j}\right) \underline{r}_{j}$. Analogously each investor cannot obtain for an asset a price greater than $\bar{r}_{j}$ and as a liability the price cannot exceed the maximum price $\left(1+h_{j}\right) \bar{r}_{j}$.

Denote the given tax rate levied on sector $i$ 's net yield on financial instrument $j$, as $\tau_{i j}$. Assume that the tax rates lie in the interval $[0,1)$ and belong to $L^{\infty}([0, T])$. Therefore, the government in this model has the flexibility of levying a distinct tax rate across both sectors and instruments.

Let us group the instrument ceiling prices $\bar{r}_{j}$ into the column vector $\bar{r}(t)=\left[\overline{r_{1}}(t), \cdots, \bar{r}_{i}(t), \cdots, \bar{r}_{n}(t)\right]^{\mathrm{T}}$, the instrument floor prices $\underline{r}_{j}$ into the column vector $\underline{r}(t)=\left[\underline{r}_{1}(t), \cdots, \underline{r}_{i}(t), \cdots, \underline{r}_{n}(t)\right]^{\mathrm{T}}$, and the tax rates $\tau_{i j}$ into the matrix

$$
\tau(t)=\left[\begin{array}{ccccc}
\tau_{11}(t) & \ldots & \tau_{1 j}(t) & \ldots & \tau_{1 n}(t) \\
\vdots & \ddots & \vdots & \ddots & \vdots \\
\tau_{i 1}(t) & \ldots & \tau_{i j}(t) & \ldots & \tau_{i n}(t) \\
\vdots & \ddots & \vdots & \ddots & \vdots \\
\tau_{m 1}(t) & \ldots & \tau_{m j}(t) & \ldots & \tau_{m n}(t)
\end{array}\right]
$$

The set of feasible instrument prices is $\mathcal{R}$.

In order to determine for each sector $i$ the optimal composition of instruments held as assets and as liabilities, we consider, as usual, the influence due to riskaversion and the process of optimization of each sector in the financial economy, namely the desire to maximize the value of the asset holdings and to minimize the value of liabilities. Then, we introduce the utility function $U_{i}\left(t, x_{i}(t), y_{i}(t), r(t)\right)$, for each sector $i$, in this way

$$
\begin{aligned}
& U_{i}\left(t, x_{i}(t), y_{i}(t), r(t)\right)=u_{i}\left(t, x_{i}(t), y_{i}(t)\right) \\
& +\sum_{j=1}^{n} r_{j}(t)\left(1-\tau_{i j}(t)\right)\left[x_{i j}(t)-\left(1+h_{j}(t)\right) y_{i j}(t)\right],
\end{aligned}
$$


where the term $-u_{i}\left(t, x_{i}(t), y_{i}(t)\right)$ represents a measure of the risk of the financial agent and

$r_{j}(t)\left(1-\tau_{i j}(t)\right)\left[x_{i}(t)-\left(1+h_{j}(t)\right) y_{i}(t)\right]$ represents the value of the difference between the asset holdings and the value of liabilities. We suppose that the sector's utility function $U_{i}\left(t, x_{i}(t), y_{i}(t)\right)$ is defined on $[0, T] \times \mathbb{R}^{n} \times \mathbb{R}^{n}$, is measurable in $t$ and is continuous with respect to $x_{i}$ and $y_{i}$. Moreover we assume that $\partial u_{i} / \partial x_{i j}$ and $\partial u_{i} / \partial y_{i j}$ exist and that they are measurable in $t$ and continuous with respect to $x_{i}$ and $y_{i}$. Further, we require that $\forall i=1, \cdots, m, \quad \forall j=1, \cdots, n$, and a.e. in $[0, T]$ the following growth conditions hold true:

$$
\left|u_{i}(t, x, y)\right| \leq \alpha_{i}(t)\|x\|\|y\|, \quad \forall x, y \in \mathbb{R}^{n},
$$

and

$$
\begin{aligned}
& \left|\frac{\partial u_{i}(t, x, y)}{\partial x_{i j}}\right| \leq \beta_{i j}(t)\|y\|, \\
& \left|\frac{\partial u_{i}(t, x, y)}{\partial y_{i j}}\right| \leq \gamma_{i j}(t)\|x\|,
\end{aligned}
$$

where $\alpha_{i}, \beta_{i j}, \gamma_{i j}$ are non-negative functions of $L^{\infty}([0, T])$. Finally, we suppose that the function

$u_{i}(t, x, y)$ is concave.

In order to determine the equilibrium prices, we establish the equilibrium condition which expresses the equilibration of the total assets, the total liabilities and the portion of financial transactions per unit $F_{j}$ employed to cover the expenses of the financial institutions including possible dividends, as in [1]. Hence, the equilibrium condition for the price $r_{j}$ of instrument $j$ is the following:

$$
\begin{gathered}
\sum_{i=1}^{m}\left(1-\tau_{i j}(t)\right)\left[x_{i j}^{*}(t)-\left(1+h_{j}(t)\right) y_{i j}^{*}(t)\right] \\
+F_{j}(t) \begin{cases}\geq 0 & \text { if } r_{j}^{*}(t)=\underline{r}_{j}(t) \\
=0 & \text { if } \underline{r}_{j}(t)<r_{j}^{*}(t)<\bar{r}_{j}(t) \\
\leq 0 & \text { if } r_{j}^{*}(t)=\bar{r}_{j}(t)\end{cases}
\end{gathered}
$$

In other words, the prices are determined taking into account the amount of the supply, the demand of an instrument and the charges $F_{j}$, namely if there is an actual supply excess of an instrument as assets and of the charges $F_{j}$ in the economy, then its price must be the floor price. If the price of an instrument is greater than $r_{j}(t)$, but not at the ceiling, then the market of that instrument must clear. Finally, if there is an actual demand excess of an instrument as liabilities and of the charges $F_{j}$ in the economy, then the price must be at the ceiling.

Now, we can give different but equivalent equilibrium conditions, each of which is useful to illustrate particular features of the equilibrium.

Definition 2.1 A vector of sector assets, liabilities and instrument prices $\left(x^{*}(t), y^{*}(t), r^{*}(t)\right) \in \prod_{i=1}^{m} P_{i} \times \mathcal{R}$ is an equilibrium of the dynamic financial model if and only if $\forall i=1, \cdots, m, \quad \forall j=1, \cdots, n$, and a.e. in $[0, T]$, it satisfies the system of inequalities

$$
\begin{aligned}
& -\frac{\partial u_{i}\left(t, x^{*}, y^{*}\right)}{\partial x_{i j}}-\left(1-\tau_{i j}(t)\right) r_{j}^{*}(t)-\mu_{i}^{(1) *}(t) \geq 0, \\
& -\frac{\partial u_{i}\left(t, x^{*}, y^{*}\right)}{\partial y_{i j}}+\left(1-\tau_{i j}(t)\right)\left(1+h_{j}(t)\right) r_{j}^{*}(t) \\
& -\mu_{i}^{(2) *}(t) \geq 0
\end{aligned}
$$

and equalities

$$
\begin{aligned}
& x_{i j}^{*}(t)\left[-\frac{\partial u_{i}\left(t, x^{*}, y^{*}\right)}{\partial x_{i j}}-\left(1-\tau_{i j}(t)\right) r_{j}^{*}(t)-\mu_{i}^{(1) *}(t)\right]=0, \\
& y_{i j}^{*}(t) \\
& \cdot\left[-\frac{\partial u_{i}\left(t, x^{*}, y^{*}\right)}{\partial x_{i j}}+\left(1-\tau_{i j}(t)\right)\left(1+h_{j}(t)\right) r_{j}^{*}(t)-\mu_{i}^{(2) *}(t)\right] \\
& =0,
\end{aligned}
$$

where $\mu_{i}^{(1) *}(t), \mu_{i}^{(2) *}(t) \in L^{2}([0, T])$ are Lagrange functions, and verify conditions (6) a.e. in $[0, T]$.

Let us explain the meaning of the above conditions. To each financial volumes $S_{i}$ and $l_{i}$ held by sector $i$, we associate the functions $\mu_{i}^{(1)}(t), \mu_{i}^{(2)}(t)$, related, respectively, to the assets and to the liabilities and which represent the "equilibrium disutilities" per unit of the sector $i$. Then, (7) and (9) mean that the financial volume invested in instrument $j$ as assets $x_{i j}^{*}$ is greater than or equal to zero if the $j$-th component

$$
-\frac{\partial u_{i}\left(t, x^{*}, y^{*}\right)}{\partial x_{i j}}-\left(1-\tau_{i j}(t)\right) r_{j}^{*}(t) \text { of the disutility is }
$$

equal to $\mu_{i}^{(1)}(t)$, whereas if

$$
-\frac{\partial u_{i}\left(t, x^{*}, y^{*}\right)}{\partial x_{i j}}-\left(1-\tau_{i j}(t)\right) r_{j}^{*}(t)>\mu_{i}^{(1)}(t) \text {, then }
$$

$x_{i j}^{*}(t)=0$. The same occurs for the liabilities and the meaning of (6) is already illustrated.

The functions $\mu_{i}^{(1)}(t)$ and $\mu_{i}^{(2)}(t)$ are Lagrange functions associated a.e. in $[0, T]$ with the constraints $\sum_{j=1}^{n} x_{i j}(t)-s_{i}(t)=0$ and $\sum_{j=1}^{n} y_{i j}(t)-l_{i}(t)=0$, respectively. They are unknown a priori, but this fact has no influence because we will prove in the following theorem that Definition is equivalent to a variational 
inequality in which $\mu_{i}^{(1)}(t)$ and $\mu_{i}^{(2)}(t)$ do not appear.

Theorem 2.1 A vector $\left(x^{*}, y^{*}, r^{*}\right) \in \prod_{i=1}^{m} P_{i} \times \mathcal{R}$ is a dynamic financial equilibrium if and only if it satisfies variational inequality (1).

Moreover, we recall the result about Lagrange multipliers (see [2]):

Theorem 2.2 Let $\left(x^{*}, y^{*}, r^{*}\right) \in \prod_{i=1}^{m} P_{i} \times L^{2}\left([0, T], \mathbb{R}_{+}^{n}\right)$ be a solution to variational inequality (1). Then there exist

$$
\begin{aligned}
& \lambda^{(1) *}, \lambda^{(2) *} \in L^{2}\left([0, T], \mathbb{R}_{+}^{m n}\right), \\
& \mu^{(1) *}, \mu^{(2) *} \in L^{2}\left([0, T], \mathbb{R}_{+}^{m}\right), \\
& \rho^{(1) *}, \rho^{(2) *} \in L^{2}\left([0, T], \mathbb{R}_{+}^{n}\right)
\end{aligned}
$$

such that a.e. in $[0, T]$,

$$
\begin{aligned}
& -\frac{\partial u_{i}\left(t, x^{*}(t), y^{*}(t)\right)}{\partial x_{i j}} \\
& -\left(1-\tau_{i j}(t)\right) r_{j}^{*}(t)-\lambda_{i j}^{(1) *}(t)-\mu_{i}^{(1) *}(t)=0, \\
& \forall i=1, \cdots, m, \forall j=1, \cdots, n, \\
& -\frac{\partial u_{i}\left(t, x^{*}(t), y^{*}(t)\right)}{\partial y_{i j}}+\left(1-\tau_{i j}(t)\right)\left(1+h_{j}(t)\right) r_{j}^{*}(t) \\
& -\lambda_{i j}^{(2) *}(t)-\mu_{i}^{(2) *}(t)=0, \\
& \forall i=1, \cdots, m, \forall j=1, \cdots, n, \\
& \sum_{i=1}^{m}\left(1-\tau_{i j}(t)\right)\left[x_{i j}^{*}(t)-\left(1+h_{j}(t)\right) y_{i j}^{*}(t)\right] \\
& +F_{j}(t)+\rho_{j}^{(2) *}(t)=\rho_{j}^{(1) *}(t), \quad \forall j=1, \cdots, n, \\
& \lambda_{i j}^{(1) *}(t) x_{i j}^{*}(t)=0, \quad \forall i=1, \cdots, m, \forall j=1, \cdots, n, \\
& \lambda_{i j}^{(2) *}(t) y_{i j}^{*}(t)=0, \\
& \mu_{i}^{(1) *}(t)\left(\sum_{j=1}^{n} x_{i j}^{*}(t)-s_{i}(t)\right)=0, \\
& \mu_{i}^{(2) *}(t)\left(\sum_{j=1}^{n} y_{i j}^{*}(t)-l_{i}(t)\right)=0, \\
& \rho_{j}^{(1) *}(t)\left(r_{j}(t)-r_{j}^{*}(t)\right)=0, \\
& \rho_{j}^{(2) *}(t)\left(r_{j}^{*}(t)-\bar{r}_{j}(t)\right)=0 .
\end{aligned}
$$

\section{Continuity Results for Financial Equilibrium Solutions}

In order to show the continuity result for the financial equilibrium problem, first of all, let us recall the wellknown property of set convergence due to K. Kuratowski (see [28]), that is a generalization of the classical Hausdorff definition of a metric for the space of closed sub- sets of a (compact) metric space.

Let $(X, d)$ be a metric space and let $\left\{K_{n}\right\}_{n \in \mathbb{N}}$ be a sequence of subsets of $X$. Recall that

$d-\underline{\lim }_{n} K_{n}=$

$\left\{x \in X: \exists\left\{x_{n}\right\}_{n \in \mathbb{N}}\right.$ eventually in $K_{n}$ such that $\left.x_{n} \stackrel{d}{\longrightarrow} x\right\}$

$d-\varlimsup_{n} K_{n}=$

$\left\{x \in X: \exists\left\{x_{n}\right\}_{n \in \mathbb{N}}\right.$ frequently in $K_{n}$ such that $\left.x_{n} \stackrel{d}{\longrightarrow} x\right\}$

where eventually means that there exists $\delta \in \mathbb{N}$ such that $x_{n} \in K_{n}$ for any $n \geq \delta$, and frequently means that there exists an infinite subset $N \subseteq \mathbb{N}$ such that $x_{n} \in K_{n}$ for any $n \in N$. Finally we can remind the set convergence in Kuratowski's sense.

Definition 3.1 We say that $\left\{K_{n}\right\}_{n \in \mathbb{N}}$ converges to some subset $K \subseteq X$ in Kuratwoski's sense, and we briefly write $K_{n} \rightarrow K$, if $d-\underline{\lim }_{n} K_{n}=d-\varlimsup_{n} K_{n}=K$. Thus, in order to verify that $K_{n} \rightarrow K$, it suffices to check that

(K1) $K \subseteq d-\underline{\lim }_{n} K_{n}$, i.e. for any $x \in K$, there exists a sequence $\left\{x_{n}\right\}_{n \in \mathbb{N}}$ converging to $x$ in $X$ such that $x_{n}$ lies in $K_{n}$ for all $n \in \mathbb{N}$;

(K2) $d-\overline{\lim }_{n} K_{n} \subseteq K$, i.e. for any subsequence $\left\{x_{n}\right\}_{n \in \mathbb{N}}$ converging to $x$ in $X$, such that $x_{n}$ lies in $K_{n}$ for all $n \in \mathbb{N}$, then the limit $x$ belongs to $K$.

Now, let us prove that the set of feasible vectors satisfies the property of the set convergence in Kuratowski's sense.

Proposition 3.1 Let $s \in C^{0}\left([0, T], \mathbb{R}^{m}\right)$, let $l \in C^{0}\left([0, T], \mathbb{R}^{m}\right)$, let $\underline{r}, \bar{r} \in C^{0}\left([0, T], \mathbb{R}_{+}^{n}\right)$ and let $\left\{t_{k}\right\}_{k \in \mathbb{N}} \subseteq[0, T]$ be a sequence such that $t_{k} \rightarrow t \in[0, T]$, as $k \rightarrow+\infty$. Then, the sequence of sets

$$
\begin{aligned}
\mathbb{K} & \left(t_{k}\right) \\
= & \left\{v\left(t_{k}\right)=\left(x\left(t_{k}\right), y\left(t_{k}\right), r\left(t_{k}\right)\right) \in \mathbb{R}^{2 m n+n}:\right. \\
& x_{i j}\left(t_{k}\right) \geq 0, y_{i j}\left(t_{k}\right) \geq 0, \forall i=1, \cdots, m, j=1, \cdots, n, \\
& \sum_{j=1}^{n} x_{i j}\left(t_{k}\right)=s_{i}\left(t_{k}\right), \sum_{j=1}^{n} y_{i j}\left(t_{k}\right)=l_{i}\left(t_{k}\right), \forall i=1, \cdots, m, \\
& \left.\underline{r}_{j}\left(t_{k}\right) \leq r_{j}\left(t_{k}\right) \leq \bar{r}_{j}\left(t_{k}\right), \forall j=1, \cdots, n\right\},
\end{aligned}
$$

$\forall k \in \mathbb{N}$, converges to

$$
\begin{aligned}
\mathbb{K}(t)= & \left\{v(t)=(x(t), y(t), r(t)) \in \mathbb{R}^{2 m n+n}:\right. \\
& x_{i j}(t) \geq 0, y_{i j}(t) \geq 0, \forall i=1, \cdots, m, j=1, \cdots, n, \\
& \sum_{j=1}^{n} x_{i j}(t)=s_{i}(t), \sum_{j=1}^{n} y_{i j}(t)=l_{i}(t), \forall i=1, \cdots, m, \\
& \left.\underline{r}_{j}(t) \leq r_{j}(t) \leq \bar{r}_{j}(t), \forall j=1, \cdots, n\right\},
\end{aligned}
$$


as $k \rightarrow+\infty$, in Kuratowski's sense.

Proof In order to prove that the sequence $\left\{\mathbb{K}\left(t_{k}\right)\right\}_{k \in \mathbb{N}}$ converges to $\mathbb{K}(t)$ in Kuratowski's sense, for any sequence $\left\{t_{k}\right\}_{n \in \mathbb{N}} \subseteq[0, T]$ such that $t_{k} \rightarrow t \in[0, T]$, as $k \rightarrow+\infty$, it is enough to show that conditions (K1) and (K2) hold.

Let $v(t)=(x(t), y(t), r(t)) \in \mathbb{K}(t)$ be fixed and let us consider the sequence

$$
\begin{array}{r}
\left\{v\left(t_{k}\right)\right\}_{k \in \mathbb{N}}=\left\{\left(x\left(t_{k}\right), y\left(t_{k}\right), r\left(t_{k}\right)\right)\right\}_{k \in \mathbb{N}}, \text { such that } \\
\forall i=1, \cdots, m, \quad \forall j=1, \cdots, n, \quad \forall k \in \mathbb{N}, \\
x_{i j}\left(t_{k}\right)=x_{i j}(t)+\frac{s_{i}\left(t_{k}\right)-s_{i}(t)}{n}, \\
y_{i j}\left(t_{k}\right)=y_{i j}(t)+\frac{l_{j}\left(t_{k}\right)-l_{j}(t)}{n}
\end{array}
$$

and $\forall j=1, \cdots, n, \forall k \in \mathbb{N}$,

$$
r_{j}\left(t_{k}\right)=\underline{r}_{j}\left(t_{k}\right)+\min \left\{r_{j}(t)-\underline{r}_{j}(t), \bar{r}_{j}\left(t_{k}\right)-\underline{r}_{j}\left(t_{k}\right)\right\} .
$$

Let us verify that $v\left(t_{k}\right) \in \mathbb{K}\left(t_{k}\right), \forall k \in \mathbb{N}$. Taking into account that

$$
\begin{aligned}
& \lim _{k \rightarrow+\infty} x_{i j}\left(t_{k}\right)=\lim _{k \rightarrow+\infty}\left(x_{i j}(t)+\frac{s_{i}\left(t_{k}\right)-s_{i}(t)}{n}\right) \\
& =x_{i j}(t) \geq 0, \\
& \lim _{k \rightarrow+\infty} y_{i j}\left(t_{k}\right)=\lim _{k \rightarrow+\infty}\left(y_{i j}(t)+\frac{l_{j}\left(t_{k}\right)-l_{j}(t)}{m}\right) \\
& =y_{i j}(t) \geq 0,
\end{aligned}
$$

there exist two index $v_{1}$ and $v_{2}$ such that for $k>v_{1}$ we get

$$
x_{i j}\left(t_{k}\right) \geq 0, \forall i=1, \cdots, m, \forall j=1, \cdots, n,
$$

and for $k>v_{2}$ we have

$$
y_{i j}\left(t_{k}\right) \geq 0, \forall i=1, \cdots, m, \forall j=1, \cdots, n .
$$

Since $\min \left\{r_{j}(t)-\underline{r}_{j}(t), \bar{r}_{j}\left(t_{k}\right)-\underline{r}_{j}\left(t_{k}\right)\right\} \geq 0$,

$\forall k \in \mathbb{N}$, it results $r_{j}\left(t_{k}\right) \geq \underline{r}_{j}\left(t_{k}\right), \forall k \in \mathbb{N}$. Moreover, being

$$
\min \left\{r_{j}(t)-\underline{r}_{j}(t), \bar{r}_{j}\left(t_{k}\right)-\underline{r}_{j}\left(t_{k}\right)\right\} \leq \bar{r}_{j}\left(t_{k}\right)-\underline{r}_{j}\left(t_{k}\right),
$$

$\forall k \in \mathbb{N}$, it follows $r_{j}\left(t_{k}\right) \leq \bar{r}_{j}\left(t_{k}\right), \forall k \in \mathbb{N}$. Finally, it results

$$
\begin{aligned}
& \lim _{k \rightarrow+\infty} r_{j}\left(t_{k}\right) \\
= & \lim _{k \rightarrow+\infty}\left[\underline{r}_{j}\left(t_{k}\right)+\min \left\{r_{j}(t)-\underline{r}_{j}(t), \bar{r}_{j}\left(t_{k}\right)-\underline{r}_{j}\left(t_{k}\right)\right\}\right] \\
= & r_{j}(t), \quad \forall j=1, \cdots, n .
\end{aligned}
$$

Then we can consider a sequence $\left\{v\left(t_{k}\right)\right\}_{k \in \mathbb{N}}$ such that for $k>v=\max \left\{v_{1}, v_{2}\right\}, \forall i=1, \cdots, m$, $\forall j=1, \cdots, n$,

$$
\begin{aligned}
& x_{i j}\left(t_{k}\right)=x_{i j}(t)+\frac{s_{i}\left(t_{k}\right)-s_{i}(t)}{n}, \\
& y_{i j}\left(t_{k}\right)=y_{i j}(t)+\frac{l_{j}\left(t_{k}\right)-l_{j}(t)}{m}, \\
& r_{j}\left(t_{k}\right)=\underline{r}_{j}\left(t_{k}\right)+\min \left\{r_{j}(t)-\underline{r}_{j}(t), \bar{r}_{j}\left(t_{k}\right)-\underline{r}_{j}\left(t_{k}\right)\right\},
\end{aligned}
$$

and for $k<v, \forall i=1, \cdots, m, \forall j=1, \cdots, n$,

$$
v\left(t_{k}\right)=P_{\mathbb{K}\left(t_{k}\right)} v(t),
$$

where $P_{\mathbb{K}\left(t_{k}\right)}(\cdot)$ denotes the Hilbertian projection on $\mathbb{K}\left(t_{k}\right)$.

We have $\lim _{n \rightarrow+\infty} v\left(t_{n}\right)=v(t)$ and for $n>v_{j}$

$$
\begin{aligned}
& \sum_{j=1}^{n} x_{i j}\left(t_{n}\right)=\sum_{j=1}^{n}\left[x_{i j}(t)+\frac{s_{i}\left(t_{k}\right)-s_{i}(t)}{n}\right]=s_{i}\left(t_{k}\right), \\
& \sum_{j=1}^{n} y_{i j}\left(t_{n}\right)=\sum_{j=1}^{n}\left[y_{i j}(t)+\frac{l_{i}\left(t_{k}\right)-l_{i}(t)}{n}\right]=l_{i}\left(t_{k}\right) .
\end{aligned}
$$

Then the first condition has been shown.

For the second one, let $\left\{v\left(t_{n}\right)\right\}_{n \in \mathbb{N}}$ be a fixed sequence, with $v\left(t_{n}\right)=\left(x\left(t_{n}\right), y\left(t_{n}\right), r\left(t_{n}\right)\right) \in \mathbb{K}\left(t_{n}\right)$,

$\forall n \in \mathbb{N}$, such that $x\left(t_{n}\right) \rightarrow x(t)$ in $\mathbb{R}^{m n}$,

$y\left(t_{n}\right) \rightarrow y(t)$ in $\mathbb{R}^{m n}, r\left(t_{n}\right) \rightarrow r(t)$ in $\mathbb{R}^{n}$. We want to prove that $v(t)=(x(t), y(t), r(t)) \in \mathbb{K}(t)$. Since

$$
\begin{gathered}
v\left(t_{n}\right)=\left(x\left(t_{n}\right), y\left(t_{n}\right), r\left(t_{n}\right)\right) \in \mathbb{K}\left(t_{n}\right), \forall n \in \mathbb{N}, \text { it results } \\
x\left(t_{n}\right) \geq 0, \forall n \in \mathbb{N}, \\
y\left(t_{n}\right) \geq 0, \forall n \in \mathbb{N}, \\
\underline{r}\left(t_{n}\right) \leq r\left(t_{n}\right) \leq \bar{r}\left(t_{n}\right), \forall n \in \mathbb{N} .
\end{gathered}
$$

Passing to the limit as $n \rightarrow+\infty$ in (15), (16) and (17), we obtain

$$
\begin{gathered}
x(t) \geq 0, \\
y(t) \geq 0, \\
\underline{r}(t) \leq r(t) \leq \bar{r}(t) .
\end{gathered}
$$

Then $v(t) \in \mathbb{K}(t)$.

The claim is, now, achieved. $\square$

For what follows, it is convenient to recall that variational inequality (1) can be rewritten in the equivalent parameterized form:

$$
\begin{aligned}
& \left\langle A\left(t, v^{*}(t)\right), v-v^{*}(t)\right\rangle \geq 0, \\
& \forall v \in \mathbb{K}(t), t \in[0, T],
\end{aligned}
$$

where the constraint set $\mathbb{K}(t), t \in[0, T]$, is a closed convex and nonempty subset of $\mathbb{R}^{n}$, $A:[0, T] \times \mathbb{R}^{2 m n+n} \rightarrow \mathbb{R}^{2 m n+n}$ is a mapping and $\langle\cdot \cdot\rangle$ denotes the scalar product in $\mathbb{R}^{n}$. Moreover, we recall that under general assumptions existence theorems have 
been proved in [2] (see Section 6).

Taking into account the general continuity result for solutions to parameter variational inequalities in reflexive Banach spaces (see [29], Theorem 4.1) and Proposition, we obtain Theorem 1.1 of Section 1.

Theorem 1.1 Let $s \in C^{0}\left([0, T], \mathbb{R}^{m}\right)$, let $l \in C^{0}\left([0, T], \mathbb{R}^{m}\right)$, let $\underline{r}, \bar{r} \in C^{0}\left([0, T], \mathbb{R}_{+}^{n}\right)$ and let $A \in C^{0}\left([0, T], \mathbb{R}^{2 m n+n}\right)$ be a strongly monotone map, namely there exists $\alpha>0$ such that, for $t \in[0, T]$,

$$
\begin{aligned}
& \left\langle A\left(t, v_{1}\right)-A\left(t, v_{2}\right), v_{1}-v_{2}\right\rangle \geq \alpha\left\|v_{1}-v_{2}\right\|^{2}, \\
& \forall v_{1}, v_{2} \in \mathbb{R}^{2 m n+n} .
\end{aligned}
$$

Then variational inequality (1) admits a unique continuous solution.

\section{Lipschitz Continuity Result}

The aim of this section is to provide a Lipschitz continuity result for the financial equilibrium solution. For this reason, we recall a general result proved in [30] for the solutions to the parameterized variational inequality (18). More precisely, the following result holds (see [30], Theorem 1):

Theorem 4.1 Let A be strongly monotone, Lipschitz continuous with respect to $v$, Lipschitz continuous with respect to $t$, and there exists $\kappa \geq 0$ such that, for $t_{1}, t_{2} \in[0, T]$,

$$
\left\|P_{\mathbb{K}\left(t_{1}\right)}(z)-P_{\mathbb{K}\left(t_{2}\right)}(z)\right\| \leq \kappa\left|t_{1}-t_{2}\right|, \forall z \in \mathbb{R}^{2 m n+n},
$$

where $P_{\mathbb{K}(t)}(z)=\arg \min _{v \in \mathbb{K}(t)}\|z-v\|, \quad t \in[0, T]$, denotes the projection onto the set $\mathbb{K}(t)$. Then, the unique solution $v^{*}(t), t \in[0, T]$, to (18) is Lipschitz continuous in $t \in[0, T], t_{1} \neq t_{2}$, the following estimate holds:

$$
\begin{aligned}
& \frac{\left\|v^{*}\left(t_{2}\right)-v^{*}\left(t_{1}\right)\right\|}{\left|t_{2}-t_{1}\right|} \\
& \leq \gamma\left(\left\|v^{*}\right\|_{C^{0}\left([0, T], \mathbb{R}^{2 m n+n}\right)}^{2}+\sup _{\substack{t_{1}, t_{2} \in[0, T] \\
t_{1} \neq t_{2}}}\left\|\frac{P_{\mathbb{K}\left(t_{2}\right)}(z)-P_{\mathbb{K}\left(t_{1}\right)}(z)}{t_{2}-t_{1}}\right\|^{2}\right)^{\frac{1}{2}},
\end{aligned}
$$

where $\gamma=\gamma(\alpha, \beta, M, T, L)$.

For the sake of simplicity, we set

$$
L=\sup _{\substack{t_{1}, t_{2} \in[0, T] \\ t_{1} \neq t_{2}}}\left\|\frac{P_{\mathbb{K}\left(t_{2}\right)}(z)-P_{\mathbb{K}\left(t_{1}\right)}(z)}{t_{2}-t_{1}}\right\| .
$$

Before applying the previous result to our dynamic financial equilibrium problem, it is necessary to estimate the variation rate of projections onto time-dependent constraint set $\left[\mathcal{P}_{\mathbb{K}}\right]$ describing the problem. It is useful to note that $\mathbb{K}(t)$ can be rewritten as the Cartesian product of the following set:

$$
\begin{aligned}
& \mathbb{K}_{1}(t)=\left\{x(t) \in \mathbb{R}^{m n}: x_{i j}(t) \geq 0, \forall i=1, \cdots, m, j=1,\right. \\
&\left.\cdots, n, \sum_{j=1}^{n} x_{i j}(t)=s_{i}(t), \forall i=1, \cdots, m\right\} \\
& \mathbb{K}_{2}(t)=\left\{y(t) \in \mathbb{R}^{m n}: y_{i j}(t) \geq 0, \forall i=1, \cdots, m, j=1,\right. \\
&\left.\cdots, n, \sum_{j=1}^{n} y_{i j}(t)=l_{i}(t), \forall i=1, \cdots, m\right\} \\
& \mathbb{K}_{3}(t)=\left\{r(t) \in \mathbb{R}^{n}: \underline{r}_{j}(t) \leq r_{j}(t) \leq \bar{r}_{j}(t), \forall j=1, \cdots, n\right\},
\end{aligned}
$$

namely

$$
\mathbb{K}(t)=\mathbb{K}_{1}(t) \times \mathbb{K}_{2}(t) \times \mathbb{K}_{3}(t) .
$$

Making use of Proposition 1 in [30], we can show that assuming $s_{i}(t), t \in[0, T]$, is Lipschitz continuous with Lipschitz constant $L_{i}^{1}$, for $i=1, \cdots, m$, it results

$$
\left\|P_{\mathbb{K}_{2}\left(t_{2}\right)}(z)-P_{\mathbb{K}_{1}\left(t_{1}\right)}(z)\right\| \leq \sqrt{n} \sum_{i=1}^{m} L_{i}^{1}\left|t_{1}-t_{2}\right| .
$$

Moreover, under the assumption that $l_{i}(t), t \in[0, T]$, is Lipschitz continuous with Lipschitz constant $L_{i}^{2}$, for $i=1, \cdots, m$, we have

$$
\left\|P_{\mathbb{K}_{2}\left(t_{2}\right)}(z)-P_{\mathbb{K}_{1}\left(t_{1}\right)}(z)\right\| \leq \sqrt{n} \sum_{i=1}^{m} L_{i}^{2}\left|t_{1}-t_{2}\right| .
$$

Now, taking into account Proposition 4.1 in [31] and assuming that $\underline{r}_{j}(t)$ and $\bar{r}_{j}(t), t \in[0, T]$, are Lipschitz continuous with Lipschitz constants $\underline{L}_{j}^{3}$ and $\overline{L_{j}^{3}}$, respectively, for $j=1, \cdots, n$, we can prove

$$
\left\|P_{\mathbb{K}_{3}\left(t_{2}\right)}(z)-P_{\mathbb{K}_{3}\left(t_{1}\right)}(z)\right\| \leq L^{3}\left|t_{1}-t_{2}\right|,
$$

where $L^{3}=\max _{j=1, \cdots, n}\left(\overline{L_{j}^{3}}+\underline{L}_{j}^{3}\right)$.

We can conclude that

Proposition 4.1 Let $t_{1}, t_{2} \in[0, T]$, let $s, l:[0, T] \rightarrow \mathbb{R}^{m}$ be two Lipschitz continuous functions and let $\underline{r}, \bar{r}:[0, T] \rightarrow \mathbb{R}^{n}$ be two Lipschitz continuous functions. Let $z$ be an arbitrary point in $\mathbb{R}^{m n}$. Then it results to be

$$
\left\|P_{\mathbb{K}_{2}\left(t_{2}\right)}(z)-P_{\mathbb{K}_{1}\left(t_{1}\right)}(z)\right\| \leq L\left|t_{2}-t_{1}\right|,
$$

where $L$ is the positive constant as in (3).

As a consequence, it results

$$
\frac{\left\|P_{\mathbb{K}\left(t_{2}\right)}(z)-P_{\mathbb{K}\left(t_{1}\right)}(z)\right\|}{\left|t_{2}-t_{1}\right|} \leq L .
$$

Hence, applying Theorem 4.1, we get the following result.

Theorem 1.2 Let $A \in C^{0}\left([0, T], \mathbb{R}^{2 m n+n}\right)$ be strongly monotone (with constant $\alpha$ ), Lipschitz continuous with 
respect to $x$ (with constant $\beta$ ), Lipschitz continuous with respect to $t$ (with constant $M$ ), and let $s, l:[0, T] \rightarrow \mathbb{R}^{m}$ be two Lipschitz continuous functions and let $\underline{r}, \bar{r}:[0, T] \rightarrow \mathbb{R}^{n}$ be two Lipschitz continuous functions. Then, the unique financial equilibrium solution $v^{*}=\left(x^{*}, y^{*}, r^{*}\right)$ is Lipschitz continuous in $[0, T]$. Moreover, let $t_{1} \neq t_{2}$, the following estimate holds:

$$
\begin{aligned}
& \frac{\left\|v^{*}\left(t_{2}\right)-v^{*}\left(t_{1}\right)\right\|^{2}}{\left|t_{2}-t_{1}\right|^{2}} \\
& \leq \gamma\left(\left\|v^{*}\right\|_{C^{0}\left([0, T], \mathbb{R}^{2 m n+n}\right)}^{2}+L^{2}\right),
\end{aligned}
$$

where $\gamma=\gamma(\alpha, \beta, M, T, L)$.

\section{Some Numerical Examples}

Let us study some numerical financial examples in which we consider as the risk aversion function the one suggested by H.M. Markowitz in [32] and [33], which expresses at each instant $t \in[0, T]$ the risk aversion by means of variance-covariance matrices denoting the sector's assessment of the standard deviation of prices for each instrument. We will see that the solutions of the examples are regular and we illustrate the impact of the components of the model on the financial equilibrium, especially for what regards $F_{1}$ and $F_{2}$ and the deficit and the surplus.

Let us consider an economy with two sectors and two financial instruments, as shown in Figure 1, but this setting is not restrictive since we can consider a general economy by an iteration of this significant case, and assume that the variance-covariance matrices of the two sectors are the following:

$$
\begin{aligned}
& Q^{1}(t)=\left[\begin{array}{cccc}
1 & 0 & -0.5 t & 0 \\
0 & 1 & 0 & 0 \\
-0.5 t & 0 & 1 & 0 \\
0 & 0 & 0 & 1
\end{array}\right], \\
& \int_{0}^{1} \sum_{i=1}^{2} \sum_{j=1}^{2}\left[2\left(\left(Q_{(11) j}^{i}(t)\right)^{T} \cdot x_{i}^{*}(t)+\left(Q_{(21) j}^{i}(t)\right)^{T} \cdot y_{i}^{*}(t)\right)-\left(1-\tau_{i j}(t)\right) r_{j}^{*}(t)\right] \\
& \times\left[x_{i j}(t)-x_{i j}^{*}(t)\right] \\
& +\sum_{i=1}^{2} \sum_{j=1}^{2}\left[2\left(\left(Q_{(12) j}^{i}(t)\right)^{T} \cdot x_{i}^{*}(t)+\left(Q_{(22) j}^{i}(t)\right)^{T} \cdot y_{i}^{*}(t)\right)+\left(1-\tau_{i j}(t)\right) r_{j}^{*}(t)\left(1+h_{j}(t)\right)\right] \\
& \times\left[y_{i j}(t)-y_{i j}^{*}(t)\right] \\
& +\sum_{j=1}^{2}\left[\sum_{i=1}^{2}\left(1-\tau_{i j}(t)\right)\left(x_{i j}^{*}(t)-\left(1+h_{j}(t)\right) y_{i j}^{*}(t)\right)+F_{j}(t)\right] \times\left[r_{j}(t)-r_{j}^{*}(t)\right] \mathrm{d} t \geq 0, \\
& \forall(x, y, r) \in \mathbb{K},
\end{aligned}
$$
work a.e. in $[0,1]$.

Let us choose as the feasible set

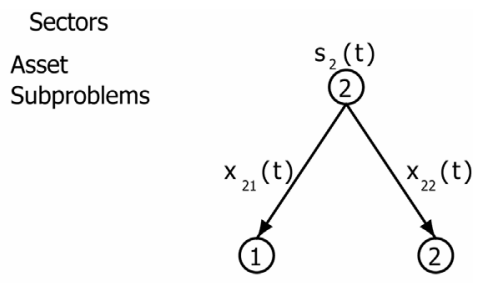

Liability
Subproblems

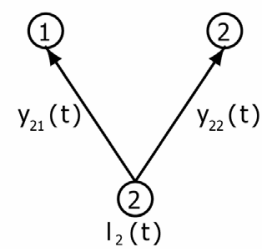

Sectors

Figure 1. Two sectors and two financial instruments net-

$$
Q^{2}(t)=\left[\begin{array}{cccc}
1 & 0 & 0 & 0 \\
0 & 1 & -0.5 t & 0 \\
0 & -0.5 t & 1 & 0 \\
0 & 0 & 0 & 1
\end{array}\right] .
$$

$$
\begin{aligned}
\mathbb{K}=\{ & \left(x_{11}(t), x_{12}(t), x_{21}(t), x_{22}(t), y_{11}(t), y_{12}(t),\right. \\
& \left.y_{21}(t), y_{22}(t), r_{1}(t), r_{2}(t)\right) \in L^{2}\left([0,1], \mathbb{R}^{10}\right): \\
& x_{11}(t)+x_{12}(t)=3 t+1, \text { a.e. in }[0,1], \\
& x_{21}(t)+x_{22}(t)=t+4, \text { a.e. in }[0,1], \\
& y_{11}(t)+y_{12}(t)=t+2, \text { a.e. in }[0,1], \\
& y_{21}(t)+y_{22}(t)=t+3, \text { a.e. in }[0,1], \\
& x_{11}(t), x_{12}(t), x_{21}(t), x_{22}(t) \geq 0, \text { a.e. in }[0,1], \\
& y_{11}(t), y_{12}(t), y_{21}(t), y_{22}(t) \geq 0, \text { a.e. in }[0,1], \\
& 0 \leq r_{1}(t) \leq 4 t+3, \text { a.e. in }[0,1], \\
& \left.0 \leq r_{2}(t) \leq 7 t+8, \text { a.e. in }[0,1]\right\} .
\end{aligned}
$$


which expresses the financial equilibrium conditions, becomes

$$
\begin{aligned}
& \int_{0}^{1}\left\{\left[2 x_{11}^{*}(t)-t y_{11}^{*}(t)-\left(1-\tau_{11}(t)\right) r_{1}^{*}(t)\right]\left(x_{11}(t)-x_{11}^{*}(t)\right)+\left[2 x_{12}^{*}(t)-\left(1-\tau_{12}(t)\right) r_{2}^{*}(t)\right]\left(x_{12}(t)-x_{12}^{*}(t)\right)\right. \\
& +\left[2 x_{21}^{*}(t)-\left(1-\tau_{21}(t)\right) r_{1}^{*}(t)\right]\left(x_{21}(t)-x_{21}^{*}(t)\right)+\left[2 x_{22}^{*}(t)-t y_{21}^{*}(t)-\left(1-\tau_{22}(t)\right) r_{2}^{*}(t)\right]\left(x_{22}(t)-x_{22}^{*}(t)\right) \\
& +\left[2 y_{11}^{*}(t)-t x_{11}^{*}(t)+\left(1+h_{1}(t)\right)\left(1-\tau_{11}(t)\right) r_{1}^{*}(t)\right]\left(y_{11}(t)-y_{11}^{*}(t)\right) \\
& +\left[2 y_{12}^{*}(t)+\left(1+h_{2}(t)\right)\left(1-\tau_{12}(t)\right) r_{2}^{*}(t)\right]\left(y_{12}(t)-y_{12}^{*}(t)\right) \\
& +\left[2 y_{21}^{*}(t)-t x_{22}^{*}(t)+\left(1+h_{1}(t)\right)\left(1-\tau_{21}(t)\right) r_{1}^{*}(t)\right]\left(y_{21}(t)-y_{21}^{*}(t)\right) \\
& +\left[2 y_{22}^{*}(t)+\left(1+h_{2}(t)\right)\left(1-\tau_{22}(t)\right) r_{2}^{*}(t)\right]\left(y_{22}(t)-y_{22}^{*}(t)\right) \\
& +\left[\left(1-\tau_{11}(t)\right) x_{11}^{*}(t)+\left(1-\tau_{21}(t)\right) x_{21}^{*}(t)-\left(1+h_{1}(t)\right)\left[\left(1-\tau_{11}(t)\right) y_{11}^{*}(t)+\left(1-\tau_{21}(t)\right) y_{21}^{*}(t)\right]+F_{1}(t)\right]\left(r_{1}(t)-r_{1}^{*}(t)\right) \\
& +\left[\left(1-\tau_{12}(t)\right) x_{12}^{*}(t)+\left(1-\tau_{22}(t)\right) x_{22}^{*}(t)-\left(1+h_{2}(t)\right)\left[\left(1-\tau_{12}(t)\right) y_{12}^{*}(t)+\left(1-\tau_{22}(t)\right) y_{22}^{*}(t)\right]+F_{2}(t)\right] \\
& \left.\left(r_{2}(t)-r_{2}^{*}(t)\right)\right\} \mathrm{d} t \geq 0, \quad \forall(x, y, r) \in \mathbb{K} .
\end{aligned}
$$

Following the direct method (see [21]) in order to find the solution, we can derive from the constraints of the convex set $\mathbb{K}$ the values of some variables in terms of the others, namely we obtain, a.e. in $[0,1]$,

$$
\begin{array}{ll}
x_{12}(t)=3 t+1-x_{11}(t), & x_{22}(t)=t+4-x_{21}(t), \\
y_{12}(t)=t+2-y_{11}(t), & y_{22}(t)=t+3-y_{21}(t) .
\end{array}
$$

Then, setting

$$
\begin{aligned}
\tilde{\mathbb{K}}=\{ & \left(x_{11}, x_{21}, y_{11}, y_{21}, r_{1}, r_{2}\right) \in L^{2}\left([0,1], \mathbb{R}^{6}\right): \\
& 0 \leq x_{11}(t) \leq 3 t+1,0 \leq x_{21}(t) \leq t+4, \text { a.e. in }[0,1] \\
& 0 \leq y_{11}(t) \leq t+2,0 \leq y_{21}(t) \leq t+3, \text { a.e. in }[0,1] \\
& \left.0 \leq r_{1}(t) \leq 4 t+3,0 \leq r_{2}(t) \leq 7 t+8, \text { a.e. in }[0,1]\right\},
\end{aligned}
$$

variational inequality (22) can be expressed in the equivalent form:

$$
\begin{aligned}
& \int_{0}^{1}\left\{\left[4 x_{11}^{*}(t)-t y_{11}^{*}(t)-\left(1-\tau_{11}(t)\right) r_{1}^{*}(t)+\left(1-\tau_{12}(t)\right) r_{2}^{*}(t)-6 t-2\right]\left(x_{11}(t)-x_{11}^{*}(t)\right)\right. \\
& +\left[4 x_{21}^{*}(t)+t y_{21}^{*}(t)-\left(1-\tau_{21}(t)\right) r_{1}^{*}(t)+\left(1-\tau_{22}(t)\right) r_{2}^{*}(t)-2 t-8\right]\left(x_{21}(t)-x_{21}^{*}(t)\right) \\
& +\left[4 y_{11}^{*}(t)-t x_{11}^{*}(t)+\left(1+h_{1}(t)\right)\left(1-\tau_{11}(t)\right) r_{1}^{*}(t)-\left(1+h_{2}(t)\right)\left(1-\tau_{12}(t)\right) r_{2}^{*}(t)-2 t-4\right]\left(y_{11}(t)-y_{11}^{*}(t)\right) \\
& +\left[t x_{21}^{*}(t)+4 y_{21}^{*}(t)+\left(1+h_{1}(t)\right)\left(1-\tau_{21}(t)\right) r_{1}^{*}(t)-\left(1+h_{2}(t)\right)\left(1-\tau_{22}(t)\right) r_{2}^{*}(t)-t^{2}-6 t-6\right]\left(y_{21}(t)-y_{21}^{*}(t)\right) \\
& +\left[\left(1-\tau_{11}(t)\right) x_{11}^{*}(t)+\left(1-\tau_{21}(t)\right) x_{21}^{*}(t)-\left(1+h_{1}(t)\right)\left(\left(1-\tau_{11}(t)\right) y_{11}^{*}(t)+\left(1-\tau_{21}(t)\right) y_{21}^{*}(t)\right)+F_{1}(t)\right]\left(r_{1}(t)-r_{1}^{*}(t)\right) \\
& +\left[-\left(1-\tau_{12}(t)\right) x_{11}^{*}(t)-\left(1-\tau_{22}(t)\right) x_{21}^{*}(t)+\left(1+h_{2}(t)\right)\left(\left(1-\tau_{12}(t)\right) y_{11}^{*}(t)+\left(1-\tau_{22}(t)\right) y_{21}^{*}(t)\right)\right. \\
& \left.\left.+\left(1-\tau_{12}(t)\right)\left(3 t+1-\left(1+h_{2}(t)\right)(t+2)\right)+\left(1-\tau_{22}(t)\right)\left[t+4-\left(1+h_{2}(t)\right)(t+3)\right]+F_{2}(t)\right]\left(r_{2}(t)-r_{2}^{*}(t)\right)\right\} \mathrm{d} t \geq 0, \\
& \quad \forall(x, y, r) \in \tilde{\mathbb{K} .}
\end{aligned}
$$

Let us set

$$
\begin{aligned}
\Gamma_{1}= & 4 x_{11}^{*}(t)-t y_{11}^{*}(t)-\left(1-\tau_{11}(t)\right) r_{1}^{*}(t)+\left(1-\tau_{12}(t)\right) r_{2}^{*}(t)-6 t-2, \\
\Gamma_{2}= & 4 x_{21}^{*}(t)+t y_{21}^{*}(t)-\left(1-\tau_{21}(t)\right) r_{1}^{*}(t)+\left(1-\tau_{22}(t)\right) r_{2}^{*}(t)-2 t-8, \\
\Gamma_{3}= & 4 y_{11}^{*}(t)-t x_{11}^{*}(t)+\left(1+h_{1}(t)\right)\left(1-\tau_{11}(t)\right) r_{1}^{*}(t)-\left(1+h_{2}(t)\right)\left(1-\tau_{12}(t)\right) r_{2}^{*}(t)-2 t-4, \\
\Gamma_{4}= & t x_{21}^{*}(t)+4 y_{21}^{*}(t)+\left(1+h_{1}(t)\right)\left(1-\tau_{21}(t)\right) r_{1}^{*}(t)-\left(1+h_{2}(t)\right)\left(1-\tau_{22}(t)\right) r_{2}^{*}(t)-t^{2}-6 t-6, \\
\Gamma_{5}= & \left(1-\tau_{11}(t)\right) x_{11}^{*}(t)+\left(1-\tau_{21}(t)\right) x_{21}^{*}(t)-\left(1+h_{1}(t)\right)\left(\left(1-\tau_{11}(t)\right) y_{11}^{*}(t)+\left(1-\tau_{21}(t)\right) y_{21}^{*}(t)\right)+F_{1}(t), \\
\Gamma_{6}= & -\left(1-\tau_{12}(t)\right) x_{11}^{*}(t)-\left(1-\tau_{22}(t)\right) x_{21}^{*}(t)+\left(1+h_{2}(t)\right)\left(\left(1-\tau_{12}(t)\right) y_{11}^{*}(t)+\left(1-\tau_{22}(t)\right) y_{21}^{*}(t)\right) \\
& +\left(1-\tau_{12}(t)\right)\left(3 t+1-\left(1+h_{2}(t)\right)(t+2)\right)+\left(1-\tau_{22}(t)\right)\left(t+4-\left(1+h_{2}(t)\right)(t+3)\right)+F_{2}(t) .
\end{aligned}
$$


As the first step of the direct method suggests, let us search solutions obtained from the system

$$
\left\{\begin{array}{l}
\Gamma_{1}=4 x_{11}^{*}(t)-t y_{11}^{*}(t)-\left(1-\tau_{11}(t)\right) r_{1}^{*}(t)+\left(1-\tau_{12}(t)\right) r_{2}^{*}(t)-6 t-2=0 \\
\Gamma_{2}=4 x_{21}^{*}(t)+t y_{21}^{*}(t)-\left(1-\tau_{21}(t)\right) r_{1}^{*}(t)+\left(1-\tau_{22}(t)\right) r_{2}^{*}(t)-2 t-8=0 \\
\Gamma_{3}=4 y_{11}^{*}(t)-t x_{11}^{*}(t)+\left(1+h_{1}(t)\right)\left(1-\tau_{11}(t)\right) r_{1}^{*}(t)-\left(1+h_{2}(t)\right)\left(1-\tau_{12}(t)\right) r_{2}^{*}(t)-2 t-4=0 \\
\Gamma_{4}=t x_{21}^{*}(t)+4 y_{21}^{*}(t)+\left(1+h_{1}(t)\right)\left(1-\tau_{21}(t)\right) r_{1}^{*}(t)-\left(1+h_{2}(t)\right)\left(1-\tau_{22}(t)\right) r_{2}^{*}(t)-t^{2}-6 t-6=0
\end{array}\right.
$$

We get the values of the variables $x_{i j}$ and $y_{i j}$ in terms of $\tau_{i j}, r_{j}$ and $h_{j}$, namely

$$
\left\{\begin{array}{l}
x_{11}^{*}(t)=\frac{1}{16-t^{2}}\left\{2 t^{2}+28 t+8+r_{1}(t)\left(1-\tau_{11}(t)\right)\left[4-t\left(1+h_{1}(t)\right)\right]+r_{2}(t)\left(1-\tau_{12}(t)\right)\left[-4+t\left(1+h_{2}(t)\right)\right]\right\}, \\
x_{12}^{*}(t)=\frac{1}{16-t^{2}}\left\{-3 t^{3}-3 t^{2}+20 t+8-r_{1}(t)\left(1-\tau_{11}(t)\right)\left[4-t\left(1+h_{1}(t)\right)\right]-r_{2}(t)\left(1-\tau_{12}(t)\right)\left[-4+t\left(1+h_{2}(t)\right)\right]\right\}, \\
x_{21}^{*}(t)=\frac{1}{16-t^{2}}\left\{-t^{3}-6 t^{2}+2 t+32+r_{1}(t)\left(1-\tau_{21}(t)\right)\left[4+t\left(1+h_{1}(t)\right)\right]-r_{2}(t)\left(1-\tau_{22}(t)\right)\left[4+t\left(1+h_{2}(t)\right)\right]\right\}, \\
x_{22}^{*}(t)=\frac{1}{16-t^{2}}\left\{2 t^{2}+14 t+32-r_{1}(t)\left(1-\tau_{21}(t)\right)\left[4+t\left(1+h_{1}(t)\right)\right]+r_{2}(t)\left(1-\tau_{22}(t)\right)\left[4+t\left(1+h_{2}(t)\right)\right]\right\}, \\
y_{11}^{*}(t)=\frac{1}{16-t^{2}}\left\{6 t^{2}+10 t+16+r_{1}(t)\left(1-\tau_{11}(t)\right)\left[t-4\left(1+h_{1}(t)\right)\right]-r_{2}(t)\left(1-\tau_{12}(t)\right)\left[t-4\left(1+h_{2}(t)\right)\right]\right\}, \\
y_{12}^{*}(t)=\frac{1}{16-t^{2}}\left\{-t^{3}-8 t^{2}+6 t+16-r_{1}(t)\left(1-\tau_{11}(t)\right)\left[t-4\left(1+h_{1}(t)\right)\right]+r_{2}(t)\left(1-\tau_{12}(t)\right)\left[t-4\left(1+h_{2}(t)\right)\right]\right\}, \\
y_{21}^{*}(t)=\frac{1}{16-t^{2}}\left\{2 t^{2}+16 t+24-r_{1}(t)\left(1-\tau_{21}(t)\right)\left[t+4\left(1+h_{1}(t)\right)\right]+r_{2}(t)\left(1-\tau_{22}(t)\right)\left[t+4\left(1+h_{2}(t)\right)\right]\right\}, \\
y_{22}^{*}(t)=\frac{1}{16-t^{2}}\left\{-t^{3}-5 t^{2}+24+r_{1}(t)\left(1-\tau_{21}(t)\right)\left[t+4\left(1+h_{1}(t)\right)\right]+r_{2}(t)\left(1-\tau_{22}(t)\right)\left[t+4\left(1+h_{2}(t)\right)\right]\right\} .
\end{array}\right.
$$

For the sake of simplicity, we assume

$$
h_{1}(t)=2 t, \quad h_{2}(t)=t
$$

We get

$$
\left\{\begin{array}{l}
x_{11}^{*}(t)=\frac{1}{16-t^{2}}\left\{2 t^{2}+28 t+8+r_{1}(t)\left(1-\tau_{11}(t)\right)\left(4-t-2 t^{2}\right)+r_{2}(t)\left(1-\tau_{12}(t)\right)\left(t^{2}+t-4\right)\right\}, \\
x_{12}^{*}(t)=\frac{1}{16-t^{2}}\left\{-3 t^{3}-3 t^{2}+20 t+8-r_{1}(t)\left(1-\tau_{11}(t)\right)\left(4-t-2 t^{2}\right)-r_{2}(t)\left(1-\tau_{12}(t)\right)\left(t^{2}+t-4\right)\right\}, \\
x_{21}^{*}(t)=\frac{1}{16-t^{2}}\left\{-t^{3}-6 t^{2}+2 t+32+r_{1}(t)\left(1-\tau_{21}(t)\right)\left(2 t^{2}+t+4\right)-r_{2}(t)\left(1-\tau_{22}(t)\right)\left(-t^{2}-t-4\right)\right\}, \\
x_{22}^{*}(t)=\frac{1}{16-t^{2}}\left\{2 t^{2}+14 t+32-r_{1}(t)\left(1-\tau_{21}(t)\right)\left(2 t^{2}+t+4\right)+r_{2}(t)\left(1-\tau_{22}(t)\right)\left(-t^{2}-t-4\right)\right\}, \\
y_{11}^{*}(t)=\frac{1}{16-t^{2}}\left\{6 t^{2}+10 t+16+r_{1}(t)\left(1-\tau_{11}(t)\right)(-7 t-4)-r_{2}(t)\left(1-\tau_{12}(t)\right)(3 t+4)\right\}, \\
y_{12}^{*}(t)=\frac{1}{16-t^{2}}\left\{-t^{3}-8 t^{2}+6 t+16-r_{1}(t)\left(1-\tau_{11}(t)\right)(-7 t-4)+r_{2}(t)\left(1-\tau_{12}(t)\right)(3 t+4)\right\}, \\
y_{21}^{*}(t)=\frac{1}{16-t^{2}}\left\{2 t^{2}+16 t+24-r_{1}(t)\left(1-\tau_{21}(t)\right)(-9 t-4)+r_{2}(t)\left(1-\tau_{22}(t)\right)(5 t+4)\right\}, \\
y_{22}^{*}(t)=\frac{1}{16-t^{2}}\left\{-t^{3}-5 t^{2}+24+r_{1}(t)\left(1-\tau_{21}(t)\right)(-9 t-4)-r_{2}(t)\left(1-\tau_{22}(t)\right)(5 t+4)\right\} .
\end{array}\right.
$$


In the following, we study various examples of financial equilibrium for which the deficit (namely $\rho_{j}^{(1) *}$ ) and surplus (namely $\rho_{j}^{(2) *}$ ) are different from zero in certain time intervals. Such examples depend on the choose of suitable values of the data. In the first example, we consider values of $\tau_{i j}$ such that

$$
\begin{aligned}
& \max \left\{\frac{22 t^{2}+27 t-4}{(4 t+3)(7 t+4)}, \frac{5 t^{3}+7 t^{2}+7 t-4}{(4 t+3)\left(2 t^{2}+t-4\right)}\right\} \leq \tau_{11}(t) \\
& \leq \min \left\{\frac{8 t^{3}+8 t^{2}-41 t-20}{(4 t+3)\left(2 t^{2}+t-4\right)}, \frac{-t^{3}+20 t^{2}+43 t+28}{(4 t+3)(7 t+4)}\right\}, \\
& \max \left\{\frac{8 t^{3}+8 t^{2}+5 t-20}{(4 t+3)\left(2 t^{2}+t+4\right)}, \frac{34 t^{2}+27 t-12}{(4 t+3)(9 t+4)}\right\} \leq \tau_{21}(t) \\
& \leq \min \left\{\frac{7 t^{3}+4 t^{2}+21 t+44}{(4 t+3)\left(2 t^{2}+t+4\right)}, \frac{-t^{3}+31 t^{2}+43 t+36}{(4 t+3)(9 t+4)}\right\},
\end{aligned}
$$

as Figures 2 and $\mathbf{3}$ show.

In particular, we assume

$$
\begin{aligned}
& \tau_{11}(t)=\frac{t+1}{2}, \quad \tau_{12}(t)=3 t, \\
& \tau_{21}(t)=t, \quad \tau_{22}(t)=\frac{5 t+1}{2} .
\end{aligned}
$$

There exist $\left.t_{1} \in\right] 0.650,0.652\left[\right.$ and $\left.t_{2} \in\right] 0.975,0.977[$, such that the vector

$$
\left\{\begin{array}{l}
x_{11}^{*}(t)=\frac{8 t^{4}+2 t^{3}-19 t^{2}+57 t+28}{2\left(16-t^{2}\right)} \\
x_{12}^{*}(t)=\frac{-8 t^{4}-8 t^{3}+17 t^{2}+39 t+4}{2\left(16-t^{2}\right)} \\
x_{21}^{*}(t)=\frac{-8 t^{4}-3 t^{3}-15 t^{2}+9 t+44}{16-t^{2}} \\
x_{22}^{*}(t)=\frac{8 t^{4}+2 t^{3}+11 t^{2}+7 t+20}{16-t^{2}} \\
y_{11}^{*}(t)=\frac{28 t^{3}+21 t^{2}-5 t+20}{2\left(16-t^{2}\right)} \\
y_{12}^{*}(t)=\frac{-30 t^{3}-25 t^{2}+37 t+44}{2\left(16-t^{2}\right)} \\
y_{21}^{*}(t)=\frac{36 t^{3}+9 t^{2}-15 t+12}{16-t^{2}} \\
y_{22}^{*}(t)=\frac{-37 t^{3}-12 t^{2}+31 t+36}{16-t^{2}} \\
r_{1}^{*}(t)=4 t+3, \\
r_{1}^{*}(t)=0,
\end{array}\right.
$$

is solution of the variational inequality in the interval ]$t_{1}, t_{2}\left[\right.$, because $x_{i j}^{*}, y_{i j}^{*}$ fulfil the constraints and

Bound function of $\tau_{11}$

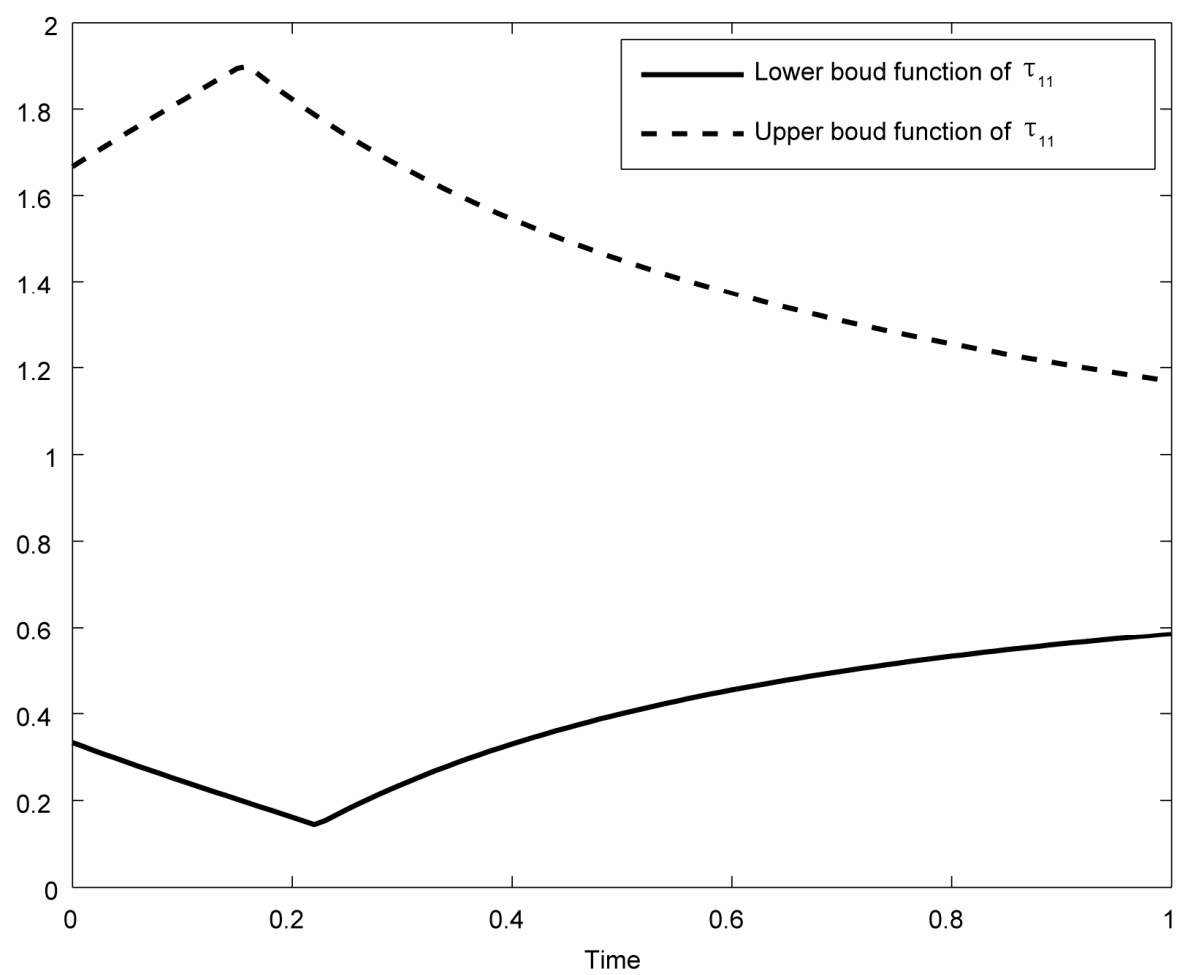

Figure 2. Bound functions of $\tau_{11}$. 


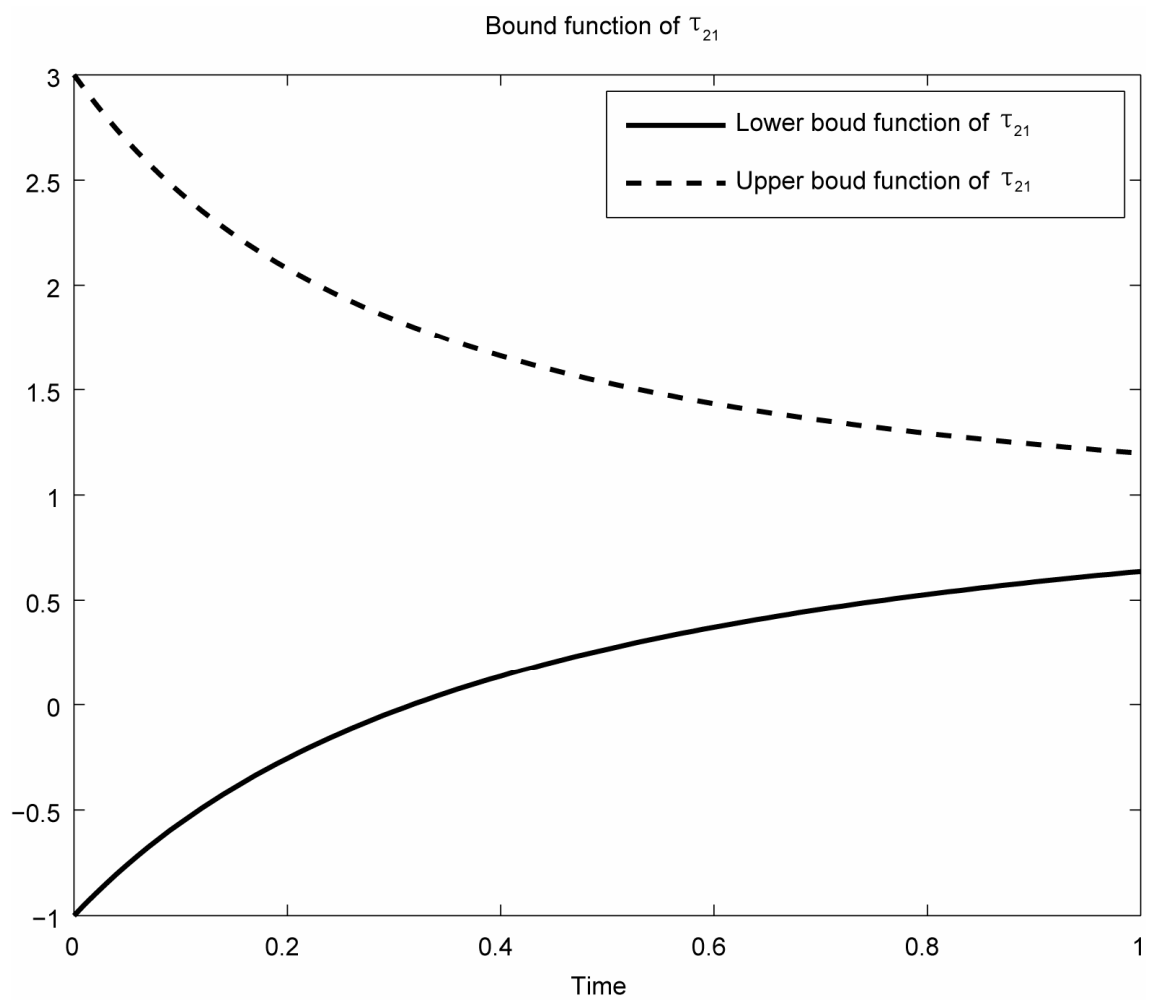

Figure 3. Bound functions of $\tau_{21}$.

$$
\begin{aligned}
& \Gamma_{5}=\frac{184 t^{5}-36 t^{4}-145 t^{3}-14 t^{2}-57 t+68}{2\left(16-t^{2}\right)}+F_{1}(t)<0, \\
& \Gamma_{6}=\frac{-291 t^{5}-329 t^{4}+123 t^{3}+423 t^{2}+98 t-56}{2\left(16-t^{2}\right)}
\end{aligned}
$$$$
+F_{2}(t)>0 \text {, }
$$

for $F_{1}(t)$ small enough and for any $F_{2}(t)$ non negative. In fact, if we consider

$$
\begin{aligned}
& \gamma_{5}=\frac{184 t^{5}-36 t^{4}-145 t^{3}-14 t^{2}-57 t+68}{2\left(16-t^{2}\right)}, \\
& \gamma_{6}=\frac{-291 t^{5}-329 t^{4}+123 t^{3}+423 t^{2}+98 t-56}{2\left(16-t^{2}\right)},
\end{aligned}
$$

we obtain that they are negative and positive respectively, as it can be verified in Figure $\mathbf{4}$ where the graphs of the numerators are represented.

Let us remember that, by virtue of Theorem 2.2, $\forall j=1,2$, the following relationships are satisfied

$$
\begin{aligned}
& \rho_{j}^{(1) *}(t)\left(\underline{r}_{j}(t)-r_{j}^{*}(t)\right)=0, \\
& \rho_{j}^{(2) *}(t)\left(r_{j}^{*}(t)-\bar{r}_{j}(t)\right)=0 ; \\
& \sum_{i=1}^{2}\left(1-\tau_{i j}(t)\right)\left[x_{i j}^{*}(t)-\left(1+h_{j}(t)\right) y_{i j}^{*}(t)\right] \\
&+F_{j}(t)+\rho_{j}^{(2) *}(t)=\rho_{j}^{(1) *}(t) .
\end{aligned}
$$

Taking into account that, in our case,

$$
\begin{gathered}
r_{1}^{*}(t)=4 t+3=\bar{r}_{1}(t), \\
r_{2}^{*}(t)=0=\underline{r}_{2}(t),
\end{gathered}
$$

from (23), we obtain

$$
\begin{aligned}
& \rho_{1}^{(1) *}(t)=0, \rho_{1}^{(2) *}(t) \geq 0, \\
& \rho_{2}^{(2) *}(t) \geq 0, \rho_{2}^{(2) *}(t)=0 .
\end{aligned}
$$

So, from (24), we get

$$
\begin{gathered}
\rho_{1}^{(2) *}(t)=-\Gamma_{5}>0, \\
\rho_{2}^{(1) *}(t)=\Gamma_{6}>0,
\end{gathered}
$$

provided that $F_{1}(t)$ is small enough and for any $F_{2}(t)$ non negative.

The importance of this example remains in the fact that, in the interval $] t_{1}, t_{2}\left[\right.$, for $F_{1}(t)$ small enough, price $r_{1}^{*}$ is maximum and the financial market has a surplus given by

$$
\begin{aligned}
& \rho_{1}^{(2) *}(t) \bar{r}_{1}(t) \\
& =-\Gamma_{5} \bar{r}_{1}(t) \\
& =\frac{-736 t^{6}-408 t^{5}+688 t^{4}+491 t^{3}+270 t^{2}-101 t-204}{2\left(16-t^{2}\right)} \\
& -F_{1}(t)(4 t+3) .
\end{aligned}
$$




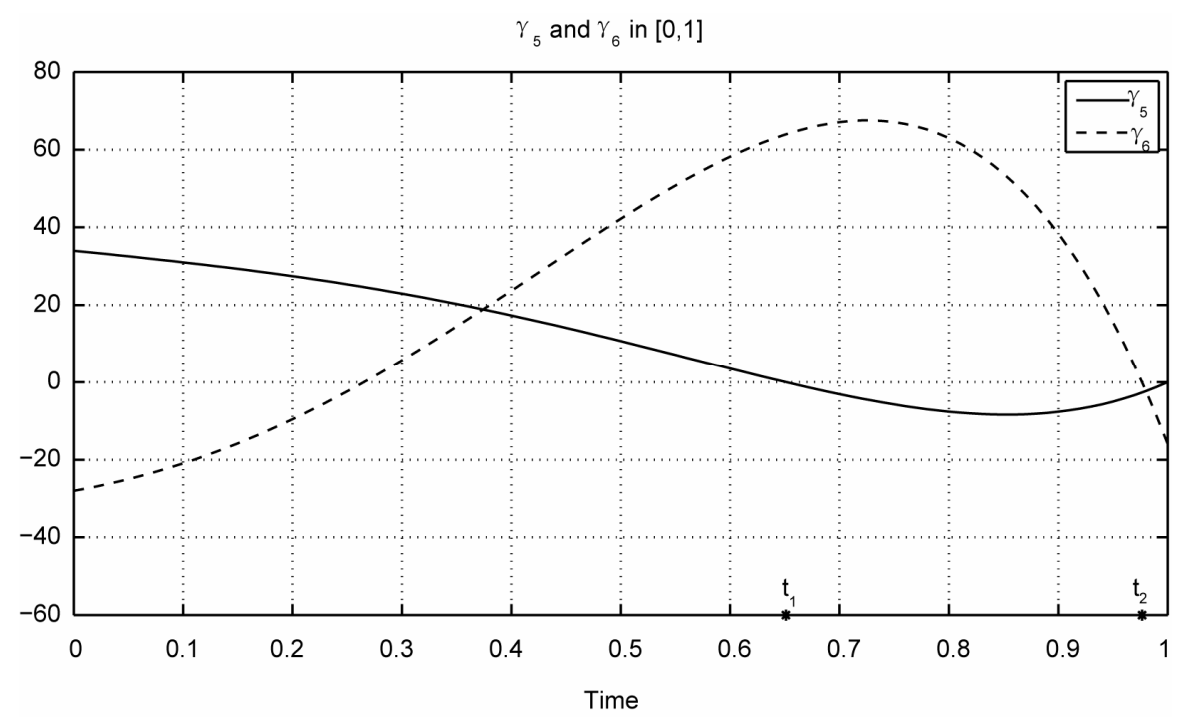

Figure 4. $\gamma_{5}$ and $\gamma_{6}$ in $[0,1]$.

Whereas, for the instrument $j=2$, we have minimal price and the deficit is given by

$$
\rho_{2}^{(1) *}(t) \underline{r}_{2}(t)=\Gamma_{6} \underline{r}_{2}(t)=0 .
$$

Now, we would like to calculate the Evaluation Index for this financial problem. More precisely, we have

$$
\begin{gathered}
\theta(t)=\frac{7 t+1}{4}, \quad i(t)=\frac{3}{2} t \\
\sum_{i=1}^{2} l_{i}(t)=2 t+5, \quad \sum_{i=1}^{m} s_{i}(t)=4 t+5 \\
\sum_{i=1}^{2} \tilde{s}_{i}(t)=\frac{\sum_{i=1}^{2} s_{i}(t)}{1+i(t)}=\frac{2(4 t+5)}{2+3 t}, \\
\sum_{j=1}^{2} \tilde{F}_{j}(t)=\frac{\sum_{j=1}^{2} F_{j}(t)}{(1+i(t))(1-\theta(t))}=\frac{8\left(F_{1}(t)+F_{2}(t)\right)}{(3-7 t)(2+3 t)} .
\end{gathered}
$$

As a consequence, the Evaluation Index is given by:

$$
\begin{aligned}
E(t) & =\frac{2 t+5}{\frac{2(4 t+5)}{2+3 t}+\frac{8\left(F_{1}(t)+F_{2}(t)\right)}{(3-7 t)(2+3 t)}} \\
& =\frac{1}{1+\frac{8\left(F_{1}(t)+F_{2}(t)\right)-t(6 t+11)(3-7 t)}{(3-7 t)(2 t+5)(2+3 t)}} .
\end{aligned}
$$

In the interval $\left[0, \frac{3}{7}[\right.$, if

$$
8\left(F_{1}(t)+F_{2}(t)\right) \leq t(6 t+11)(3-7 t)
$$

the economy has a positive average evaluation. The same situation happens if

$$
8\left(F_{1}(t)+F_{2}(t)\right) \geq t(6 t+11)(3-7 t)
$$

in the interval $\left.] \frac{3}{7}, 1\right]$ (where $3-7 t<0$ ).

Now let us consider the case where the values of $\tau_{i j}$ are such that

$$
\begin{aligned}
& \max \left\{\frac{7 t^{3}+17 t^{2}+8 t-24}{(7 t+8)\left(t^{2}+t-4\right)}, \frac{t^{3}+29 t^{2}+46 t+16}{(7 t+8)(3 t+4)}\right\} \leq \tau_{12}(t) \\
& \leq \min \left\{\frac{10 t^{3}+18 t^{2}-40 t-40}{(7 t+8)\left(t^{2}+t-4\right)}, \frac{27 t^{2}+62 t+48}{(7 t+8)(3 t+4)}\right\}, \\
& \max \left\{\frac{8 t^{3}+21 t^{2}+34 t}{(7 t+8)\left(t^{2}+t+4\right)}, \frac{t^{3}+40 t^{2}+68 t+8}{(7 t+8)(5 t+4)}\right\} \leq \tau_{22}(t) \\
& \leq \min \left\{\frac{7 t^{3}+17 t^{2}+50 t+64}{(7 t+8)\left(t^{2}+t+4\right)}, \frac{37 t^{2}+84 t+56}{(7 t+8)(5 t+4)}\right\},
\end{aligned}
$$

as Figures 5 and 6 show.

In particular, we assume

$$
\begin{aligned}
& \tau_{11}(t)=3 t, \quad \tau_{12}(t)=-\frac{1}{5} t+\frac{6}{5} \\
& \tau_{21}(t)=t, \quad \tau_{22}(t)=-\frac{1}{5} t+\frac{6}{5} .
\end{aligned}
$$

There exist $\left.t_{3} \in\right] 0.946,0.948\left[\right.$ and $\left.t_{4} \in\right] 0.997,0.999[$, such that the vector 


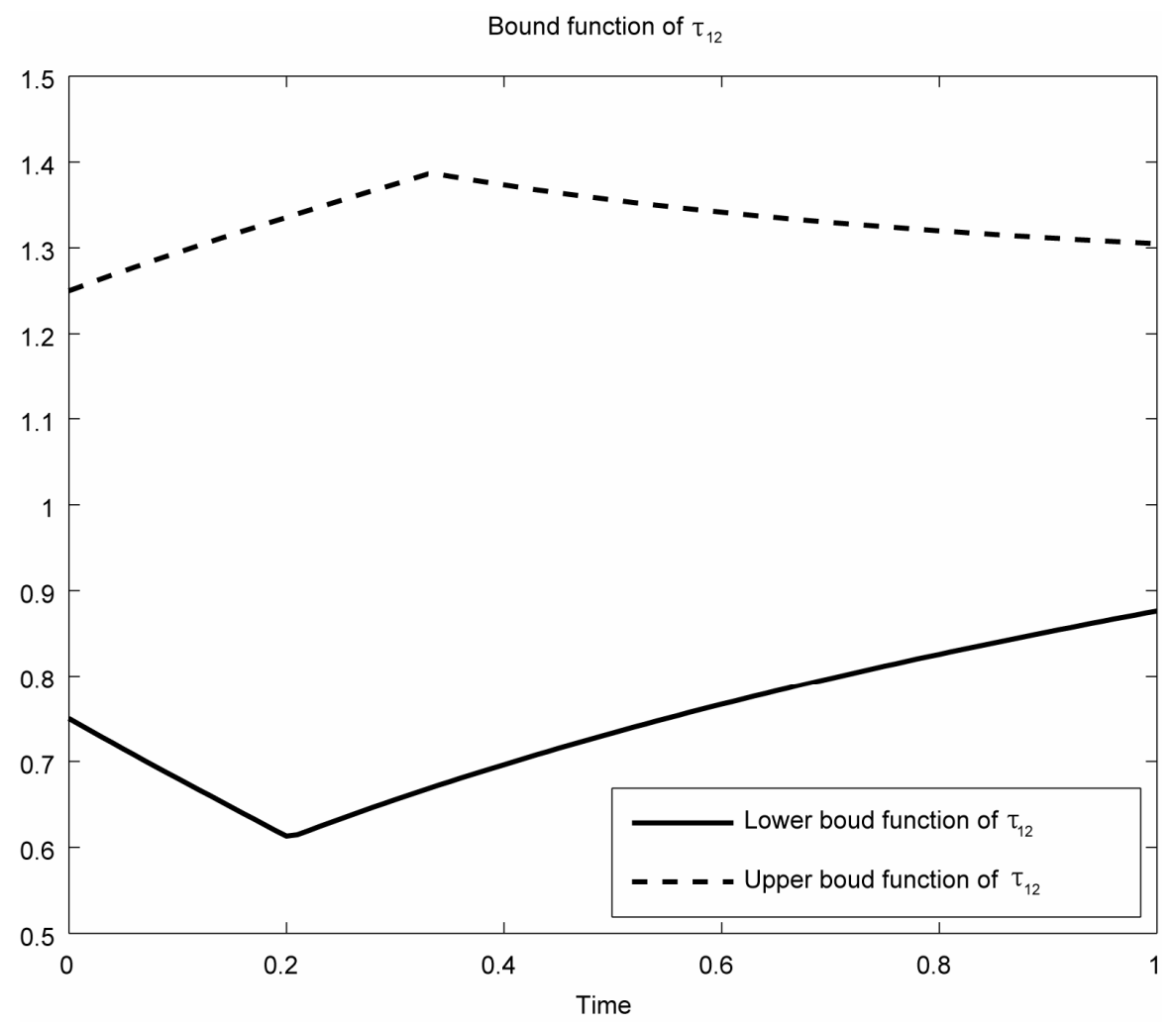

Figure 5. $\gamma_{5}$ and $\gamma_{6}$ in $[0,1]$.

Bound function of $\tau_{22}$

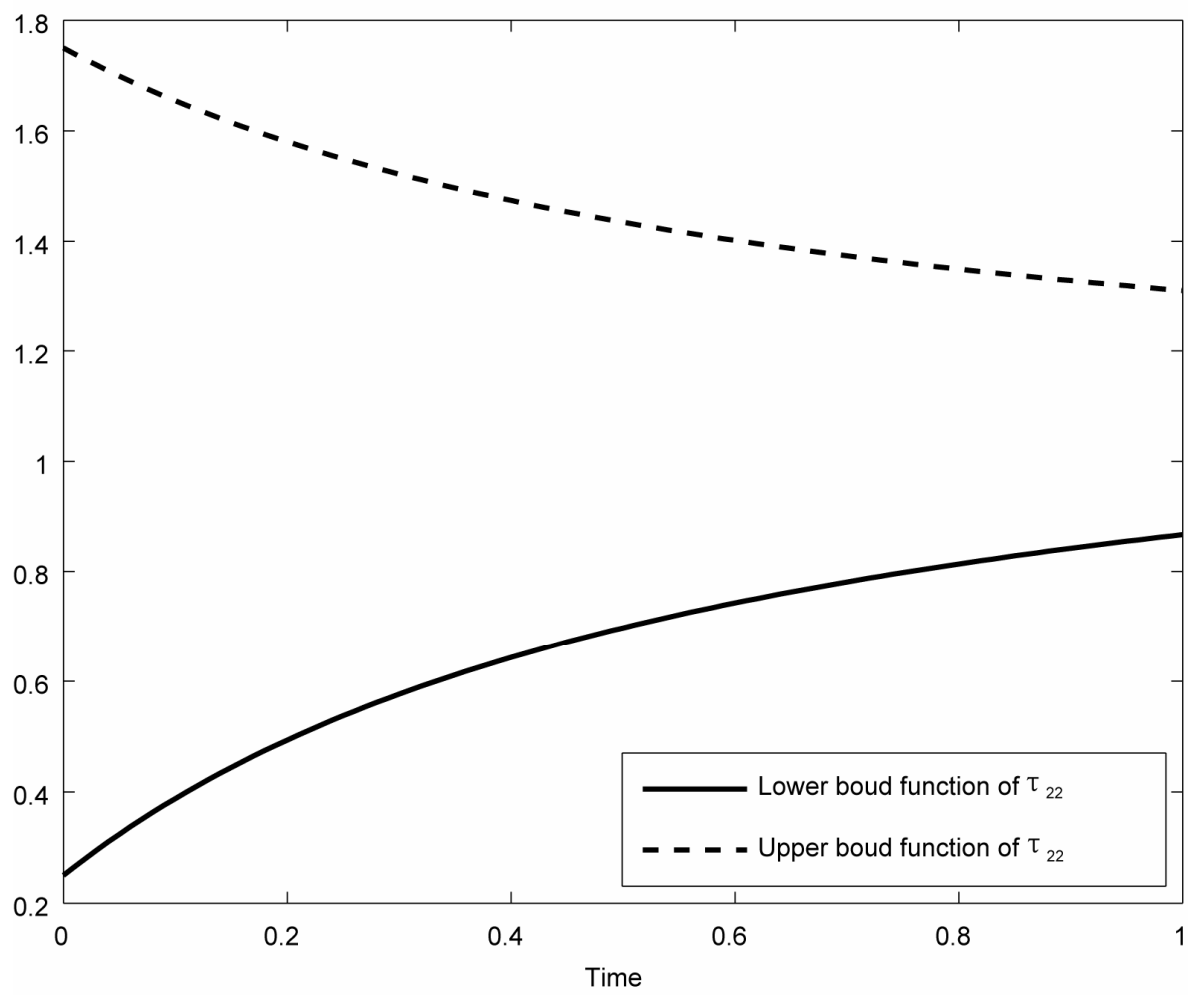

Figure 6. $\gamma_{5}$ and $\gamma_{6}$ in $[0,1]$. 


$$
\left\{\begin{array}{l}
x_{11}^{*}(t)=\frac{7 t^{4}+8 t^{3}-25 t^{2}+128 t+72}{5\left(16-t^{2}\right)} \\
x_{12}^{*}(t)=\frac{-7 t^{4}-23 t^{3}+20 t^{2}+112 t+8}{5\left(16-t^{2}\right)} \\
x_{21}^{*}(t)=\frac{-7 t^{4}-13 t^{3}-51 t^{2}+14 t+192}{5\left(16-t^{2}\right)} \\
x_{22}^{*}(t)=\frac{7 t^{4}+8 t^{3}+31 t^{2}+66 t+128}{5\left(16-t^{2}\right)} \\
y_{11}^{*}(t)=\frac{21 t^{3}+61 t^{2}+30 t+48}{5\left(16-t^{2}\right)} \\
y_{12}^{*}(t)=\frac{-26 t^{3}-71 t^{2}+50 t+62}{5\left(16-t^{2}\right)} \\
y_{21}^{*}(t)=\frac{35 t^{3}+43 t^{2}+44 t+88}{5\left(16-t^{2}\right)} \\
r_{12}^{*}(t)=0, \\
r_{2}^{*}(t)=7 t+8, \\
5\left(16-t^{2}\right)
\end{array}\right.
$$

is solution of the variational inequality in the interval ]$t_{3}, t_{4}\left[\right.$, because $x_{i j}^{*}, y_{i j}^{*}$ fulfil the constraints and

$\Gamma_{5}=\frac{182 t^{5}+427 t^{4}+351 t^{3}-128 t^{2}-380 t+128}{5\left(16-t^{2}\right)}$

$+F_{1}(t)>0$

$\Gamma_{6}=\frac{66 t^{5}+114 t^{4}-86 t^{3}-266 t^{2}+44 t+128}{25\left(16-t^{2}\right)}+F_{2}(t)<0$, for any $F_{1}(t)$ non negative and for $F_{2}(t)$ small enough. In fact, if we consider

$$
\begin{aligned}
& \gamma_{5}=\frac{182 t^{5}+427 t^{4}+351 t^{3}-128 t^{2}-380 t+128}{5\left(16-t^{2}\right)}, \\
& \gamma_{6}=\frac{66 t^{5}+114 t^{4}-86 t^{3}-266 t^{2}+44 t+128}{25\left(16-t^{2}\right)},
\end{aligned}
$$

we obtain that they are positive and negative respectively, as it can be verified in Figures $\mathbf{7}$ and $\mathbf{8}$ where the graphs of the numerators are represented in detail.

If we remember that, in this case,

$$
\begin{gathered}
r_{1}^{*}(t)=0=\underline{r}_{1}(t), \\
r_{2}^{*}(t)=7 t+8=\bar{r}_{2}(t),
\end{gathered}
$$

from (23), we obtain

$$
\begin{aligned}
& \rho_{1}^{(1) *}(t) \geq 0, \quad \rho_{1}^{(2) *}(t)=0, \\
& \rho_{2}^{(1) *}(t)=0, \rho_{2}^{(2) *}(t) \geq 0 .
\end{aligned}
$$

So, from (24), we get

$$
\begin{gathered}
\rho_{1}^{(1) *}(t)=\Gamma_{5}>0, \\
\rho_{2}^{(2) *}(t)=-\Gamma_{6}>0,
\end{gathered}
$$

provided that $F_{2}(t)$ is small enough and for any $F_{1}(t)$ non negative.

In this situation, we can assert that, in the interval ]$t_{3}, t_{4}[$, for the instrument $j=1$, we have minimal price and the deficit is given by

$$
\rho_{1}^{(1) *}(t) \underline{r}_{1}(t)=\Gamma_{5} \underline{r}_{1}(t)=0 .
$$

Whereas, for $F_{2}(t)$ small enough, price $r_{2}^{*}$ is maximum and the financial market has a surplus given by

$$
\rho_{2}^{(2) *}(t) \bar{r}_{2}(t)=-\Gamma_{6} \bar{r}_{2}(t)=\frac{-462 t^{6}-605 t^{5}+514 t^{4}+2550 t^{3}+1820 t^{2}-1248 t-1024}{25\left(16-t^{2}\right)}-F_{2}(t)(7 t+8)
$$

Now let us proceed to the calculation of the Evaluation Index. We have

$$
\begin{aligned}
& \theta(t)=\frac{9 t+6}{10}, \quad i(t)=\frac{3}{2} t, \\
& \sum_{i=1}^{2} l_{i}(t)=2 t+5, \\
& \sum_{i=1}^{m} S_{i}(t)=4 t+5, \\
& \sum_{i=1}^{2} \tilde{S}_{i}(t)=\frac{\sum_{i=1}^{2} S_{i}(t)}{1+i(t)}=\frac{2(4 t+5)}{2+3 t},
\end{aligned}
$$

$\sum_{j=1}^{2} \tilde{F}_{j}(t)=\frac{\sum_{j=1}^{2} F_{j}(t)}{(1+i(t))(1-\theta(t))}=\frac{20\left(F_{1}(t)+F_{2}(t)\right)}{(4-9 t)(2+3 t)}$.

Then the Evaluation Index is

$$
\begin{aligned}
E(t) & =\frac{2 t+5}{\frac{2(4 t+5)}{2+3 t}+\frac{20\left(F_{1}(t)+F_{2}(t)\right)}{(4-9 t)(2+3 t)}} \\
& =\frac{1}{1+\frac{20\left(F_{1}(t)+F_{2}(t)\right)-t(6 t+11)(4-9 t)}{(4-9 t)(2+3 t)(2 t+5)}} .
\end{aligned}
$$




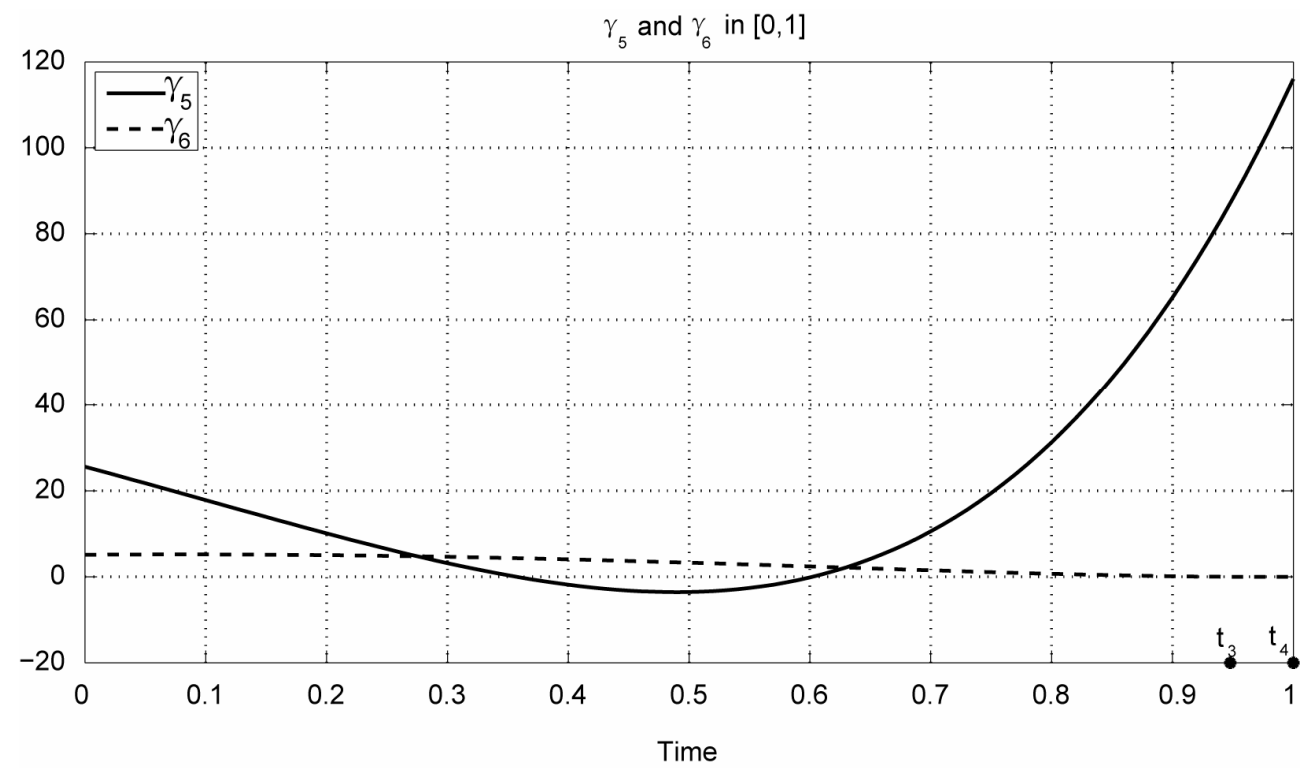

Figure 7. $\gamma_{5}$ and $\gamma_{6}$ in $[0,1]$.

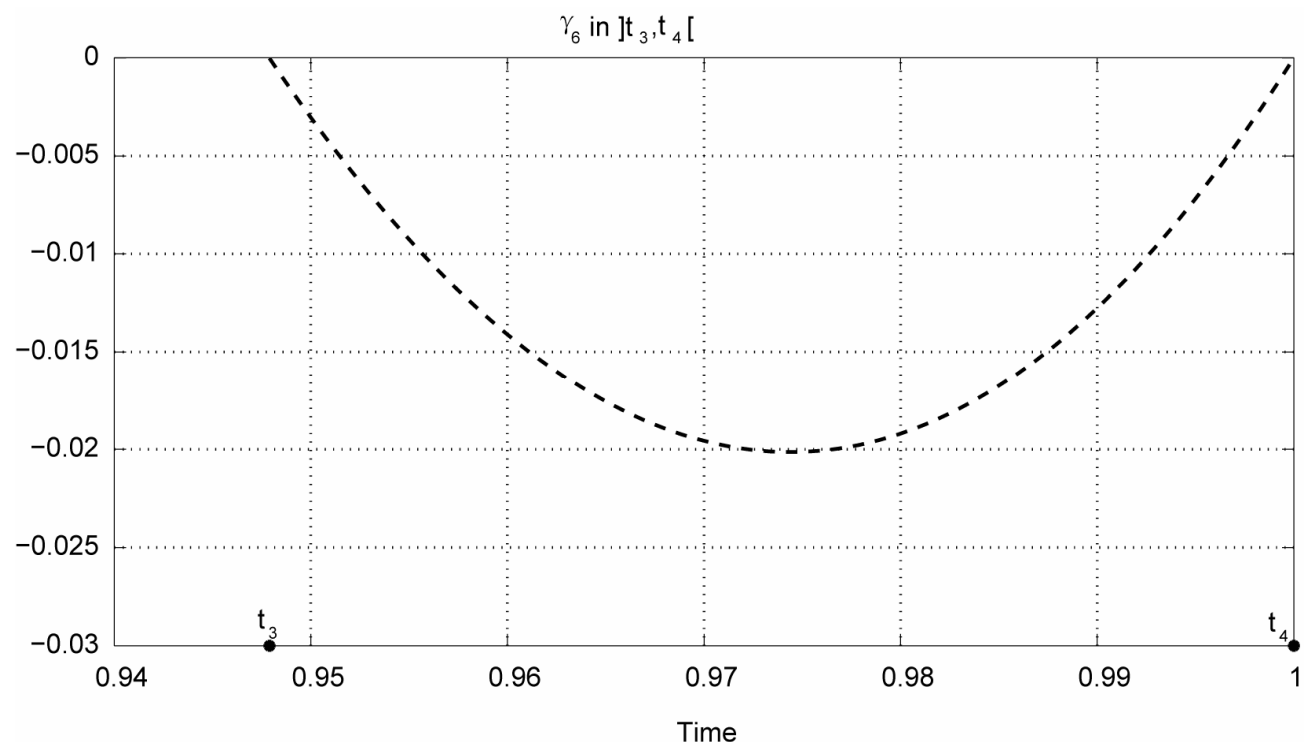

Figure 8. $\gamma_{5}$ and $\gamma_{6}$ in $[0,1]$.

As a consequence, in the interval $\left[0, \frac{4}{9}[\right.$, if

$$
20\left(F_{1}(t)+F_{2}(t)\right) \leq t(6 t+11)(4-9 t)
$$

the economy has a positive average evaluation. The same situation happens if

$$
20\left(F_{1}(t)+F_{2}(t)\right) \geq t(6 t+11)(4-9 t)
$$

in the interval $\left.] \frac{4}{9}, 1\right]$ (where $\left.4-9 t<0\right)$. From this example we see that we can get useful information from the $E(t)$, which requires simple calculations.

Now, we would like to study another example in which we assume

$$
\begin{gathered}
\tau_{11}(t)=3 t, \quad \tau_{12}(t)=-t+1, \\
\tau_{21}(t)=t, \quad \tau_{22}(t)=-\frac{1}{2} t+\frac{1}{2} .
\end{gathered}
$$

Under these assumptions, there exist $\left.t_{5} \in\right] 0,638 ; 0,640\left[\right.$ and $\left.t_{6} \in\right] 0,997 ; 0,999[$, such that the vector 


$$
\left\{\begin{array}{l}
x_{11}^{*}(t)=\frac{2 t^{2}+28 t+8}{16-t^{2}}, \\
x_{12}^{*}(t)=\frac{-3 t^{3}-3 t^{2}+20 t+8}{16-t^{2}} \\
x_{21}^{*}(t)=\frac{-t^{3}-6 t^{2}+2 t+32}{16-t^{2}}, \\
x_{22}^{*}(t)=\frac{2 t^{2}+14 t+32}{16-t^{2}} \\
y_{11}^{*}(t)=\frac{6 t^{2}+10 t+16}{16-t^{2}} \\
y_{12}^{*}(t)=\frac{-t^{3}-8 t^{2}+6 t+16}{16-t^{2}} \\
y_{21}^{*}(t)=\frac{2 t^{2}+16 t+24}{16-t^{2}} \\
y_{22}^{*}(t)=\frac{-t^{3}-5 t^{2}+24}{16-t^{2}}, \\
r_{1}^{*}(t)=0 \\
r_{2}^{*}(t)=0
\end{array}\right.
$$

is solution of the variational inequality in the interval ]$t_{5}, t_{6}\left[\right.$, because $x_{i j}^{*}, y_{i j}^{*}$ fulfil the constraints and

$$
\begin{aligned}
& \Gamma_{5}=\frac{41 t^{4}+95 t^{3}+40 t^{2}-60 t}{16-t^{2}}+F_{1}(t)>0, \\
& \Gamma_{6}=\frac{3 t^{5}+19 t^{4}+11 t^{3}-7 t^{2}-18 t+8}{2\left(16-t^{2}\right)}+F_{2}(t)>0,
\end{aligned}
$$

for $F_{1}(t)$ and $F_{2}(t)$ non negative. In fact, if we consider

$$
\begin{aligned}
& \gamma_{5}=\frac{41 t^{4}+95 t^{3}+40 t^{2}-60 t}{16-t^{2}}, \\
& \gamma_{6}=\frac{3 t^{5}+19 t^{4}+11 t^{3}-7 t^{2}-18 t+8}{2\left(16-t^{2}\right)},
\end{aligned}
$$

we obtain that they are both positive, as it can be verified in Figure 9 where the graphs of the numerators are represented.

We observe that, in our case

$$
\begin{aligned}
& r_{1}^{*}(t)=0=\underline{r}_{1}(t), \\
& r_{2}^{*}(t)=0=\underline{r}_{2}(t),
\end{aligned}
$$

so, from (23), it follows that

$$
\begin{aligned}
& \rho_{1}^{(1) *}(t) \geq 0, \quad \rho_{1}^{(2) *}(t)=0, \\
& \rho_{2}^{(1) *}(t) \geq 0, \rho_{2}^{(2) *}(t)=0 .
\end{aligned}
$$

Then, from (24), we obtain

$$
\begin{aligned}
& \rho_{1}^{(1) *}(t)=\Gamma_{5}>0, \\
& \rho_{2}^{(1) *}(t)=\Gamma_{6}>0,
\end{aligned}
$$

for any $F_{1}(t)$ and $F_{2}(t)$ non negative.

As consequence of the meaning of $\rho_{j}^{(i) *}$, in the interval $] t_{5}, t_{6}[$, for both the instruments, we have minimal price and the deficit is given, respectively, by

$$
\begin{aligned}
& \rho_{1}^{(1) *}(t) \underline{r}_{1}(t)=\Gamma_{5} \underline{r}_{1}(t)=0, \\
& \rho_{2}^{(2) *}(t) \underline{r}_{2}(t)=\Gamma_{6} \underline{r}_{1}(t)=0 .
\end{aligned}
$$

As regards the calculation of the Evaluation Index, we have

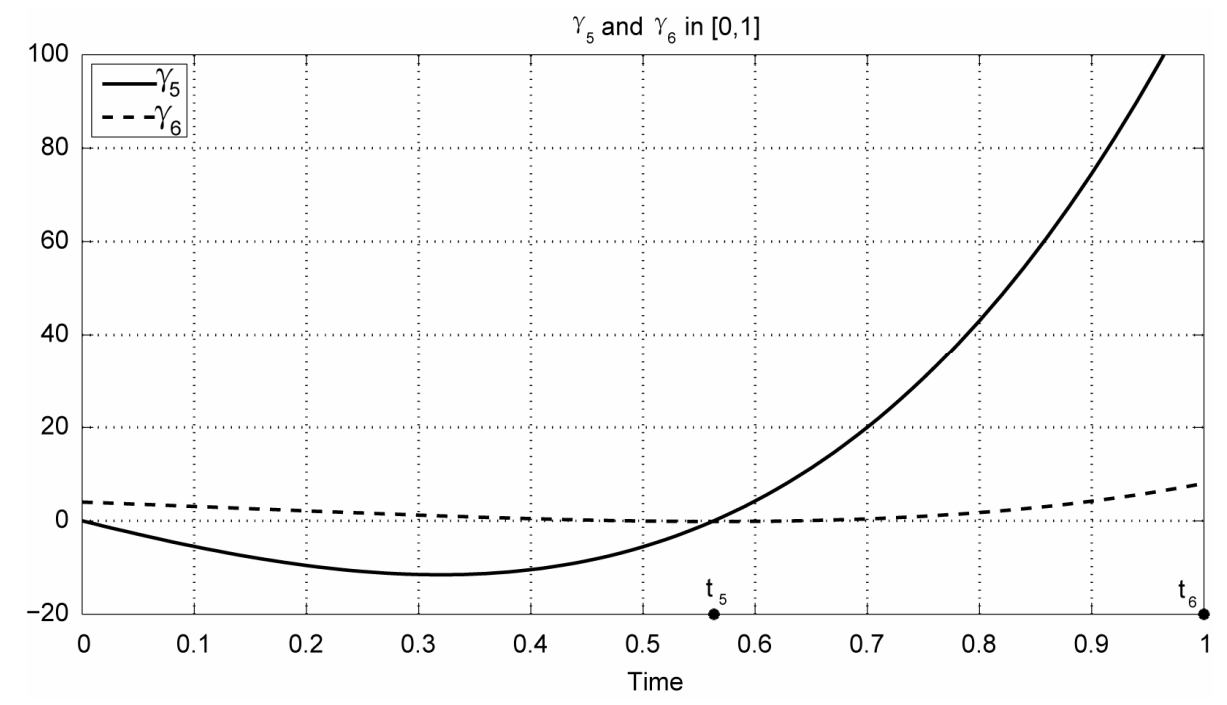

Figure 9. $\gamma_{5}$ and $\gamma_{6}$ in $[0,1]$. 


$$
\begin{aligned}
& \theta(t)=\frac{5 t+3}{8}, \quad i(t)=\frac{3}{2} t \\
& \sum_{i=1}^{2} l_{i}(t)=2 t+5, \quad \sum_{i=1}^{m} s_{i}(t)=4 t+5 \\
& \sum_{i=1}^{2} \tilde{s}_{i}(t)=\frac{\sum_{i=1}^{2} s_{i}(t)}{1+i(t)}=\frac{2(4 t+5)}{2+3 t}, \\
& \sum_{j=1}^{2} \tilde{F}_{j}(t)=\frac{\sum_{j=1}^{2} F_{j}(t)}{(1+i(t))(1-\theta(t))}=\frac{16\left(F_{1}(t)+F_{2}(t)\right)}{5(1-t)(2+3 t)} .
\end{aligned}
$$

As a consequence, the Evaluation Index is

$$
\begin{aligned}
E(t) & =\frac{2 t+5}{\frac{2(4 t+5)}{2+3 t}+\frac{16\left(F_{1}(t)+F_{2}(t)\right)}{5(1-t)(2+3 t)}} \\
& =\frac{1}{1+\frac{16\left(F_{1}(t)+F_{2}(t)\right)-\frac{1}{6} t(6 t+11)(1-t)}{5(1-t)(2+3 t)(2 t+5)}} .
\end{aligned}
$$

Then, in the interval $[0,1[$, if

$$
16\left(F_{1}(t)+F_{2}(t)\right) \leq \frac{1}{6} t(6 t+11)(1-t)
$$

the economy has a positive average evaluation because $1-t<0$. From this example we see that it is easier to achieve financial balance if $\tau_{i j}$ and $h_{j}$ are more or less equal.

\section{REFERENCES}

[1] A. Barbagallo, P. Daniele and A. Maugeri, "Variational Formulation for a General Dynamic Financial Equilibrium Problem. Balance Law and Liability Formula," Nonlinear Analysis: Theory, Methods \& Applications, Vol. 75, No. 3, 2012, pp. 1104-1123. doi:10.1016/i.na.2010.10.013

[2] A. Barbagallo, P. Daniele, S. Giuffrè and A. Maugeri, "Variational Approach for a General Financial Equilibrium Problem: the Deficit Formula, the Balance Law and the Liability Formula, a Path to the Economy Recover," Submitted.

[3] A. Barbagallo, "Degenerate Time-Dependent Variational Inequalities with Applications to Traffic Equilibrium Problems," Computational Methods in Applied Mathematics, Vol. 6, No. 2, 2006, pp. 117-133.

[4] A. Barbagallo, "Regularity Results for Time-Dependent Variational and Quasi-Variational Inequalities and Application to Calculation of Dynamic Traffic Network," Mathematical Models and Methods in Applied Sciences, Vol. 17, No. 2, 2007, pp. 277-304. doi:10.1142/S0218202507001917

[5] A. Barbagallo, "Regularity Results for Evolutionary Non- linear Variational and Quasi-Variational Inequalities with Applications to Dynamic Equilibrium Problems," Journal of Global Optimization, Vol. 40, No. 1-3, 2008, pp. 29-39. doi:10.1007/s10898-007-9194-5

[6] A. Barbagallo, "Existence and Regularity of Solutions to Nonlinear Degenerate Evolutionary Variational Inequalities with Applications to Traffic Equilibrium Problem," Applied Mathematics and Computation, Vol. 208, No. 1, 2009, pp. 1-13. doi:10.1016/j.amc.2008.10.030

[7] A. Barbagallo, "On the Regularity of Retarded Equilibria in Time-Dependent Traffic Equilibrium Problems," Nonlinear Analysis, Vol. 71, No. 12, 2009, pp. e2406-e2417. doi:10.1016/j.na.2009.05.054

[8] P. Daniele and S. Giuffrè, "General Infinite Dimensional Duality and Applications to Evolutionary Network Equilibrium Problems," Optimization Letters, Vol. 1, No. 3, 2007, pp. 227-243.

[9] P. Daniele, S. Giuffré and A. Maugeri, "Remarks on General Infinite Dimensional Duality with Cone and Equality Constraints," Communications in Applied Analysis, Vol. 13, No. 4, 2009, pp. 567-578.

[10] P. Daniele, S. Giuffrè, G. Idone and A. Maugeri, "Infinite Dimensional Duality and Applications," Mathematische Annalen, Vol. 339, No. 1, 2007, pp. 221-239. doi:10.1007/s00208-007-0118-y

[11] A. Maugeri and F. Raciti, "Remarks on Infinite Dimensional Duality," Journal of Global Optimization, Vol. 46, No. 4, 2010, pp. 581-588. doi:10.1007/s10898-009-9442-y

[12] M. B. Donato, M. Milasi and C. Vitanza, "Dynamic Walriasian Price Equilibrium Problem: Evolutionary Variational Approach with Sensitivity Analysis," Optimization Letters, Vol. 2, No. 1, 2008, pp. 113-126. doi:10.1007/s11590-007-0047-4

[13] M. B. Donato, M. Milasi and C. Vitanza, "Quasi-Variational Approach of a Competitive Economic Equilibrium Problem with Utility Function: Existence of Equilibrium," Mathematical Models and Methods in Applied Sciences, Vol. 18, No. 3, 2008, pp. 351-367. doi:10.1142/S0218202508002711

[14] M. B. Donato, A. Maugeri, M. Milasi and C. Vitanza, "Duality Theory for a Dynamic Walrasian Pure Exchange Economy," Pacific Journal of Optimization, Vol. 4, No. 3, 2008, pp. 537-547.

[15] M. B. Donato, M. Milasi and C. Vitanza, "A New Contribution to a Dynamic Competitive Equilibrium Problem," Applied Mathematics Letters, Vol. 23, No. 2, 2010, pp. 148-151. doi:10.1016/j.aml.2009.09.002

[16] A. Barbagallo and M.-G. Cojocaru, "Dynamic Equilibrium Formulation of Oligopolistic Market Problem," Mathematical and Computer Modelling, Vol. 49, No. 5-6, 2009, pp. 966-976. doi:10.1016/j.mcm.2008.02.003

[17] A. Barbagallo and A. Maugeri, "Duality Theory for the Dynamic Oligopolistic Market Equilibrium Problem," Optimization, Vol. 60, No. 1-2, 2011, pp. 29-52. doi:10.1080/02331930903578684

[18] S. Giuffrè and S. Pia, "Weighted Traffic Equilibrium Problem in Non Pivot Hilbert Spaces," Nonlinear Analy- 
sis, Vol. 71, No. 12, 2009, pp. e2054-e2061. doi:10.1016/j.na.2009.03.044

[19] A. Barbagallo and S. Pia, "Weighted Variational Inequalities in Non-Pivot Hilbert Spaces with Applications," Computational Optimization and Applications, Vol. 48, No. 3, 2011, pp. 487-514. doi:10.1007/s10589-009-9259-0

[20] S. Giuffrè, G. Idone and S. Pia, "Some Classes of Projected Dynamical Systems in Banach Spaces and Variational Inequalities," Journal of Global Optimization, Vol. 40, No. 1-3, 2008, pp. 119-128. doi:10.1007/s10898-007-9173-x

[21] A. Maugeri, "Convex Programming, Variational Inequalities and Applications to the Traffic Equilibrium Problem," Applied Mathematics \& Optimization, Vol. 16, No. 2, 1987, pp. 169-185. doi:10.1007/BF01442190

[22] P. Daniele, "Variational Inequalities for Evolutionary Financial Equilibrium," In: A. Nagurney, Ed., Innovations in Financial and Economic Networks, Edward Elgar Publishing, Cheltenham, 2003, pp. 84-108.

[23] P. Daniele, "Variational Inequalities for General Evolutionary Financial Equilibrium," In: F. Giannessi and A. Maugeri, Eds., Variational Analysis and Applications, Springer Verlag, New York, 2005, pp. 279-299.

[24] P. Daniele, "Evolutionary Variational Inequalities Applied to Financial Equilibrium Problems in an Environment of Risk and Uncertainty," Nonlinear Analysis, Vol. 63, No. 5-7, 2005, pp. 1645-1653.

[25] P. Daniele, "Dynamic Networks and Evolutionary Variational Inequalities," Edward Elgar Publishing, Chentelham, 2006.
[26] P. Daniele, "Evolutionary Variational Inequalities and Applications to Complex Dynamic Multi-Level Models," Transportation Research Part E, Vol. 46, No. 6, 2010, pp. 855-880. doi:10.1016/j.tre.2010.03.005

[27] P. Daniele, S. Giuffrè and S. Pia, "Competitive Financial Equilibrium Problems with Policy Interventions," Journal of Industrial and Management Optimization, Vol. 1, No. 1, 2005, pp. 39-52. doi:10.3934/jimo.2005.1.39

[28] K. Kuratowski, “Topology,” Academic Press, New York, 1966.

[29] A. Barbagallo and M.-G. Cojocaru, "Continuity of Solutions for Parametric Variational Inequalities in Banach Space," Journal of Mathematical Analysis and Applications, Vol. 351, No. 2, 2009, pp. 707-720. doi:10.1016/j.jmaa.2008.10.052

[30] A. Maugeri and L. Scrimali, "Global Lipschitz Continuity of Solutions to Parameterized Variational Inequalities," Bollettino Unione Matematica Italiana, Vol. 2, No. 9, 2009, pp. 45-69.

[31] A. Barbagallo and R. Di Vincenzo, "Lipschitz Continuity and Duality for Dynamic Oligopolistic Market Equilibrium Problem with Memory Term," Journal of Mathematical Analysis and Applications, Vol. 382, No. 1, 2011, pp. 231-247. doi:10.1016/j.jmaa.2011.04.042

[32] H. M. Markowitz, "Portfolio Selection," The Journal of Finance, Vol. 7, No. 1, 1952, pp. 77-91.

[33] H. M. Markowitz, "Portfolio Selection: Efficient Diversification of Investments," Wiley, New York, 1959. 